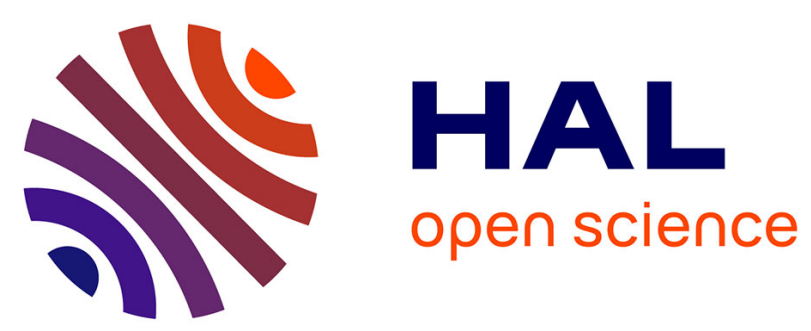

\title{
Prevention against equality?
}

Marc Fleurbaey, Grégory Ponthière

\section{To cite this version:}

Marc Fleurbaey, Grégory Ponthière. Prevention against equality?. 2012. halshs-00677393

\section{HAL Id: halshs-00677393 \\ https://shs.hal.science/halshs-00677393}

Preprint submitted on 8 Mar 2012

HAL is a multi-disciplinary open access archive for the deposit and dissemination of scientific research documents, whether they are published or not. The documents may come from teaching and research institutions in France or abroad, or from public or private research centers.
L'archive ouverte pluridisciplinaire HAL, est destinée au dépôt et à la diffusion de documents scientifiques de niveau recherche, publiés ou non, émanant des établissements d'enseignement et de recherche français ou étrangers, des laboratoires publics ou privés. 


\section{PARISSCHOQL OF ECQNOMICS}

WORKING PAPER N²012 - 12

Prevention against equality?

Marc Fleurbaey

Grégory Ponthière

JEL Codes: D63, D71, I12, J18

Keywords: Compensation, Equality, Longevity, Mortality, Prevention

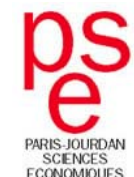




\title{
Prevention Against Equality?
}

\author{
Marc FLEURBAEY* Gregory PONTHIERE ${ }^{\dagger}$
}

March 7, 2012

\begin{abstract}
Common sense supports prevention policies aimed at improving survival prospects among the population. It is also widely acknowledged that an early death is a serious disadvantage, and that attention should be paid to the compensation of short-lived individuals. This paper re-examines the compatibility of those two concerns: prevention against early death and compensation for early death. We show that, under mild conditions, no social ordering on allocations can satisfy a concern for prevention and a concern for compensation. The reason is that if it is socially desirable to raise the number of survivors through prevention, it must also be, under costly prevention, desirable to deteriorate the living standards of the short-lived. We then explore two approaches to the prevention / compensation dilemma, and study the associated optimal allocation of resources.

Keywords: compensation, equality, longevity, mortality, prevention.

JEL codes: D63, D71, I12, J18.
\end{abstract}

*Princeton University.

${ }^{\dagger}$ Ecole Normale Superieure, Paris - Paris School of Economics. [corresponding author] Address: Ecole Normale Supérieure, 48 boulevard Jourdan, building B, 2nd floor, Office B, 75014 Paris, France. E-mail: gregory.ponthiere@ens.fr Telephone: 0033143136204. 


\section{Introduction}

Death is the unique certain event of every human life. That unique sure thing gives rise, at the individual level, to three major types of reactions. A first reaction is to try to forget death. A second reaction is to try to postpone death as much as possible. A third reaction consists of trying to make death as benign as possible, by reducing the disadvantage associated to it.

At the social level, the first reaction seems hardly reasonable: one would certainly not like a society that treats its citizens as if these were invulnerable. On the contrary, one may expect from a fair or a good society to do as much as possible to postpone the death of its citizens, and to make their death as benign as possible. Those two requirements invite public policies of various kinds.

On the one hand, the desire to postpone death invites massive prevention programs against premature death. Although longevity inequalities are largely due to factors on which individuals have little control (e.g. genes, pollution, early childhood), it remains true that longevity is partly endogenous: a person can, to some extent, influence his life expectancy by adopting more or less healthy lifestyles. ${ }^{1}$ Hence there is a strong support for prevention policies diffusing the healthy ways of life. In the light of the significant impact of lifestyles on mortality, such policies could have a large impact. For instance, Balia and Jones (2008), in their study on premature mortality in Great Britain, correcting for biases due to endogeneity and unobserved heterogeneity, find that lifestyles predict about 25 percent of the overall inequality in mortality, with strong contributions of smoking and sleep patterns. ${ }^{2}$

On the other hand, there also exists a strong support for making death as benign as possible. A premature death is a serious disadvantage, which gives rise to large inequalities in well-being across individuals. From the point of view of social justice, such inequalities are unacceptable, and there is a strong appeal for compensating the short-lived, by reducing the disadvantage due to death as much as possible. Compensating the short-lived seems, at first glance, infeasible, since short-lived persons can be neither identified ex ante nor affected ex post (after their longevity is revealed). However, as shown by Fleurbaey et al (2011), such a compensation can be carried out under general conditions, by concentrating the consumption of resources on the early ages of life. ${ }^{3}$ Replacing smoothed or increasing consumption profiles by decreasing consumption profiles reduces the disadvantage due to early death.

Those two concerns - i.e. postponing death and making it benign - are both intuitive, and their corollaries in terms of policies are in conformity with common sense. A massive prevention against early death and the compensation of the short-lived seem to be equally desirable. From the perspective of social justice,

\footnotetext{
${ }^{1}$ On the impact of genes on longevity inequalities, see Christensen et al (2006).

${ }^{2}$ Other studies on the same topic include: Auster et al (1969), Mullahy and Portney (1990), Mullahy and Sindelar (1996), and Contoyannis and Jones (2004).

${ }^{3}$ The compensation proposed by Fleurbaey et al (2011) follows the lines of the egalitarianequivalent approach to social justice (discussed below). Note that such a policy requires the knowledge, by the government, of the statistical distribution of the age at death.
} 
those two concerns both favour equality, but on different grounds. Massive prevention favours the equality of longevities within the population, while the compensation of the short-lived favours the equality of standards of living among agents with unequal longevities. Egalitarians should thus want them both.

However, the main finding of this paper is that there is tension between the goal to postpone death as much as possible for everyone, and the goal to make death as benign as possible for everyone. The reason for this tension is that prevention may impose costs on all individuals and ultimately harm those who die early in spite of sharing in the cost. We therefore explore the compatibility between prevention against early death and compensation for early death.

To study this issue, we consider a pure exchange economy with a finite population of finitely-lived individuals. In this economy there is a risk on the length of life, and individuals can raise their life expectancy through healthimproving efforts (e.g. jogging). In this framework, we examine the possibility to construct a social ordering on the set of all possible allocations (i.e. lifetime consumption and prevention efforts profiles for all agents), on the basis of several axioms capturing our concerns for prevention and compensation.

Note that such a framework, although simple, captures two important features of real life. First, the model emphasizes that the possibility to influence one's life expectancy does not coincide with a perfect control on actual longevity. This is in conformity with empirical studies showing that lifestyles affect survival prospects on average, but that adopting a particular lifestyle does not guarantee a certain longevity, since longevity remains inherently risky. Second, our framework allows for the heterogeneity of individual preferences on (un)healthy lifestyles. This heterogeneity in attitudes toward lifestyles is also in conformity with real life. It is, for instance, a matter of fact that some persons like having physical activities, whereas other persons hate this kind of activity. But given that those heterogeneous preferences generate inequalities in life expectancies and, possibly, in actual longevities, they can hardly be ignored.

In this framework, the concern for prevention takes the form of a simple condition, the Survivors Numbers Count axiom, which states that, as soon as it delivers a strictly longer life to some individuals (other things equal), a small enough rise in the prevention effort made by all individuals is socially desirable. The concern for compensation, on the other hand, take the form of an axiom called Pigou-Dalton for Equal Preferences, Efforts and Lifetimes (PDEPEL). This axiom says that if we consider two agents who have the same longevity and the same preferences, then a transfer from the richer agent to the poorer agent constitutes a social improvement (everything else being unchanged).

Anticipating on our results, we show that, under mild conditions, such as the Weak Pareto axiom and the Hansson Independence axiom, there exists no social ordering on allocations satisfying both the Survivors Numbers Count axiom and PDEPEL. In other words, it is impossible to satisfy both our concern for prevention against early death and our concern for compensation for early death. The reason is that if it is socially desirable to raise the number of survivors through prevention (as implied by Survivors Numbers Count), it must also be socially desirable, if prevention is costly, to deteriorate the living standards of 
the unlucky short-lived, which goes against compensation and equality concerns (and thus against our Pigou-Dalton axiom). Hence attempts to postpone death as much as possible conflicts with attempts to make early death as benign as possible.

Therefore some choice has to be made between those two natural reactions in front of death. This paper explores two approaches to such dilemma.

A first approach consists of giving the priority to compensation, by letting the Survivors Numbers Count axiom aside. It can then be shown that, provided we add another version of the Pigou-Dalton Principle (i.e., the Pigou-Dalton Principle for Constant Consumption and Reference Effort and Lifetime - PDCCREL), the social ordering on allocations must satisfy the maximin property on a particular index of well-being that we call the Constant Consumption Profile Equivalent for Reference Lifetime and Effort (CCPERLE). That is, the approach evaluates a particular social state by looking at the smallest consumption the agents would accept in the replacement of their current situation, if they could benefit from longevity and efforts of reference.

A second approach consists of giving the priority to prevention, by letting PDEPEL aside. Actually, the set of social ordering functions satisfying Weak Pareto, Hansson Independence, Survivors Numbers Count and PDCCREL is not empty, and within the class of social ordering functions satisfying those axioms, there is a salient family of social ordering functions, which consists in the social ordering functions involving a continuous and quasi-concave social welfare function applied to CCPERLE levels.

Having derived these two alternative approaches to the dilemma between prevention and compensation, we will then explore the precise form of the social optimum in a simple 2-period economy where longevities are unequal, and where agents, who differ in time preferences and in preferences towards effort, can affect their life expectancy through dedication to prevention effort. For this purpose, we start considering a first-best setting, in which individual preferences can be observed by the social planner, but not individual longevities - only the statistical distribution of deaths for all effort levels is known ex ante. Then, we also consider a second-best setting, in which individual preferences cannot be observed.

The rest of this paper is organized as follows. Section 2 presents the framework. Section 3 presents plausible ethical axioms accounting for the prevention and the compensation concerns, and shows that no social objective can satisfy those axioms. Section 4 studies two approaches to the dilemma: priority to compensation or priority to prevention. Section 5 examines the corresponding social optima under a full observability of agents' preferences. Section 6 considers some extensions, including the second-best problem. Section 7 concludes.

\section{The framework}

The model describes the situation of a given finite population of agents with ordinal preferences over lifetime consumption and dedication profiles. We consider 
a pure exchange economy.

The set of natural integers (resp., real numbers) is denoted $\mathbb{N}$ (resp., $\mathbb{R}$ ). Let $N$ be the set of individuals, with cardinality $|N|$. The maximum possible lifespan for any individual, i.e., the maximum number of periods that can ever be lived, is denoted by $T$, with $T \in \mathbb{N}$ and $T>1$.

Each individual will have a particular lifetime consumption profile. Under the assumption of non-negative consumptions, a lifetime consumption profile for an individual $i \in N$ is a vector of dimension $T$ or less.

Each individual also has a particular lifetime preventive effort profile. Under the assumption of non-negative effort, a lifetime effort profile for an individual $i \in N$ is a vector of dimension $T$ or less. ${ }^{4}$

For the simplicity of presentation, let us call the pair $\left(c_{i}, e_{i}\right)$ the life of individual $i$, and denote this life by $x_{i}=\left(c_{i}, e_{i}\right)$. Following this notation, the set of all possible (individual) lives can be denoted by $X=\bigcup_{\ell=1}^{T}\left(\mathbb{R}_{+}^{\ell} \times \mathbb{R}_{+}^{\ell}\right)$.

The longevity of an individual $i$ with life $x_{i}$ is defined by a function $\lambda$ : $X \rightarrow \mathbb{N}$, such that $\lambda\left(x_{i}\right)$ is the dimension of the lifetime consumption and effort profiles, that is, the length of existence of individual $i$.

An allocation defines a consumption profile $c_{i}$ and a health effort profile $e_{i}$ for each individual in $N$. An allocation for $N$ can also be written as a list of lives for all members of $N$, that is, a vector $x_{N}:=\left(x_{i}\right)_{i \in N} \in X^{|N|}$. We denote by $\left.x_{i}\right|_{\ell}$ the beginning of life of sublength $\ell$ of individual $i$ with longevity $\lambda\left(x_{i}\right)>\ell$. That part of life includes a consumption subprofile, denoted by $\left.c_{i}\right|_{\ell}$, and an effort subprofile, denoted by $\left.e_{i}\right|_{\ell}$.

Each individual $i \in N$ has well-defined preferences over the set of all possible lives $X$. His preferences are described by an ordering $R_{i}$ (i.e. a reflexive, transitive and complete binary relation). For all $x_{i} \in X$, the indifference set at $x_{i}$ for $R_{i}$ is defined as $I\left(x_{i}, R_{i}\right):=\left\{x_{i}^{\prime} \in X \mid x_{i}^{\prime} I_{i} x_{i}\right\}$. For any lives $x_{i}$ and $x_{i}^{\prime}$ of equal length, preference orderings on $x_{i}$ and $x_{i}^{\prime}$ are assumed to be continuous, convex and weakly monotonic in consumption (i.e. if we consider $\left(c_{i}, e_{i}\right)$ and $\left(c_{i}^{\prime}, e_{i}^{\prime}\right), c_{i} \geq c_{i}^{\prime}$ and $e_{i}=e_{i}^{\prime}$ imply $x_{i} R_{i} x_{i}^{\prime}$, and $c_{i} \gg c_{i}^{\prime}$ and $e_{i}=e_{i}^{\prime}$ imply $\left.x_{i} P_{i} x_{i}^{\prime}\right)$. Note that we do not assume here monotonicity of preferences with respect to health-improving effort, that is, we leave open the possibility to have some individuals who like making health-improving efforts, whereas other individuals dislike this kind of practice. However, we assume that for all lives $x_{i} \in X$, there exists a life $x$ with full length and with constant consumption $(c, \ldots, c) \in \mathbb{R}_{+}^{T}$ and effort $(e, \ldots, e) \in \mathbb{R}_{+}^{T}$ such that $x_{i} I_{i} x$, which means that no life is worse or better than all lives with full longevity. This excludes lexicographic preferences for which longevity is an absolute good or bad. Let $\Re$ be the set of all preference orderings on $X$ satisfying these properties. A preference profile for $N$ is a list of preference orderings of the members of $N$, denoted $R_{N}:=\left(R_{i}\right)_{i \in N} \in \Re^{|N|}$.

Finally, it should be stressed here that the preferences of the agent, as described by the ordering $R_{i}$ on $X$, are assumed to be respectable preferences,

\footnotetext{
${ }^{4}$ Note that using such an effort variable is a simplification. One could, instead, have a monetary effort (entering the budget constraint) or a temporal effort (entering the time constraint). We use a purely physical effort on the grounds of analytical simplicity.
} 
in the sense that these are the preferences to be taken into account when assessing the goodness and the fairness of allocations. Clearly, in the context of health-affecting choices, the occurrence of regrets is widespread, and such regrets reveal the existence of a tension between, on the one hand, the preferences that governed the choices of agents (i.e. the ex ante preferences), and, on the other hand, the preferences that agents use when evaluating their lives (i.e. the ex post preferences). ${ }^{5}$ The existence of such a dual self raises the question of the preferences to be taken into account in social valuations. Usually, there is a tendency to consider that the most respectable preferences are the ones observed towards the end of life, life being regarded as a period of learning about oneself. Our approach will follow that standard view and take the ex post preferences of agents into account, and not the ex ante preferences.

\section{The social objective}

The goal of this section is to examine the possibility to derive a social objective that does justice to the prevention against an early death and to the compensation for an early death. For that purpose, we will, in a first stage, present some basic ethical axioms that social preferences ought to satisfy. Then, in a second stage, we will propose two axioms that account for the ideas of preventing early death within the population and compensating short-lived persons.

Social preferences will be formalized by a social ordering function $\succsim$ which associates every admissible preference profile $R_{N}$ of the population with an ordering $\succsim_{R_{N}}$ defined on the set of all possible allocations for $N$, that is, an ordering defined on $X^{|N|}$. For all $x_{N}, x_{N}^{\prime} \in X^{|N|}, x_{N} \gtrsim_{R_{N}} x_{N}^{\prime}$ means that the allocation $x_{N}$ is, under the preference profile $R_{N}$, at least as good as the allocation $x_{N}^{\prime}$. The symbols $\succ_{R_{N}}$ and $\sim_{R_{N}}$ will denote strict preference and indifference, respectively.

A first, standard axiom imposed on social preferences consists of the Weak Pareto axiom, which states that if all individuals prefer one allocation to another, then this should also be regarded as socially preferable to that alternative.

Axiom 1 (Weak Pareto) For all preference profiles $R_{N} \in \Re^{|N|}$, all allocations $x_{N}, x_{N}^{\prime} \in X^{|N|}$, if $x_{i} P_{i} x_{i}^{\prime}$ for all $i \in N$, then $x_{N} \succ_{R_{N}} x_{N}^{\prime}$.

This axiom can be justified as a guarantee against the choice of inefficient allocations, or, alternatively, as a way to insure the respect for individual preferences. In the context of health-affecting choices, the reliance on the Pareto axiom is quite liberal, since individual preferences may go against behaviors maximizing individual health.

\footnotetext{
${ }^{5}$ For instance, Slovic (2001) found, on the basis of a telephone survey of a representative sample of U.S. respondents, that $85 \%$ of adult smokers stated that they would not start smoking if they had to do it over again. That result is robust to various countries. In the U.K., Jarvis et al (2002) showed that about $83 \%$ of smokers "would not start smoking if they had their time again". Finally, Fong et al (2004) showed, on the basis of a telephone survey in Canada, the U.S., the U.K. and Australia, that about $90 \%$ of smokers agree with the statement "if you had to do it over again, you would not have started smoking"
} 
In the social choice literature, it is also common, in order to avoid Arrowtype impossibility results, to impose the Hansson Independence axiom. That condition, which was first introduced by Hansson (1973) and Pazner (1979), requires that social preferences over two allocations depend only on the individual indifference curves at these allocations.

Axiom 2 (Hansson Independence) For all preferences profiles $R_{N}, R_{N}^{\prime} \in$ $\Re^{|N|}$ and for all allocations $x_{N}, x_{N}^{\prime} \in X^{|N|}$, if for all $i \in N, I\left(x_{i}, R_{i}\right)=I\left(x_{i}, R_{i}^{\prime}\right)$ and $I\left(x_{i}^{\prime}, R_{i}\right)=I\left(x_{i}^{\prime}, R_{i}^{\prime}\right)$, then $x_{N} \succsim_{R_{N}} x_{N}^{\prime}$ if and only if $x_{N} \succsim_{R_{N}^{\prime}} x_{N}^{\prime}$.

In comparison to Arrow's Independence of Irrelevant Alternatives, Hansson Independence is much weaker, since the indifference curves at the allocations under consideration contain more information than individual pairwise preferences over these two allocations. This is what preserves us from Arrow's impossibility.

Let us now formulate, in the context of our economy, a concern for postponing death as much as possible. Such concern for prevention can take the form of the Survivors Numbers Count axiom. This axiom states that, as soon as it guarantees a strictly longer life to one person (and the same longevities for all others), a general increase in preventive effort is socially desirable.

Axiom 3 (Survivors Numbers Count) For all $R_{N} \in \Re^{|N|}$, all $x_{N}, x_{N}^{\prime} \in$ $X^{|N|}$, all $i \in N$, if $\lambda\left(x_{i}\right)<\lambda\left(x_{i}^{\prime}\right)$, if $x_{i}=\left.x_{i}^{\prime}\right|_{\lambda\left(x_{i}\right)}, x_{j}=x_{j}^{\prime}$ for all $j \neq i$, and if $x_{i}^{\prime} P_{i} x_{i}$, then there exists a vector of effort $e_{N}^{\prime \prime} \gg e_{N}^{\prime}$ such that:

$$
\left(c_{i}^{\prime}, e_{i}^{\prime \prime}\right)_{i \in N} \succsim_{R_{N}}\left(x_{i}\right)_{i \in N}
$$

The Survivors Numbers Count axiom states that the certainty of lengthening the life of one person justifies, from a social perspective, a rise in the levels of individual health efforts. This condition is quite intuitive, since the increase in preventive effort is not based here on the expectation of saving one life (as in policy decisions), but, rather, on the absolute certainty to save one life. ${ }^{6}$

Regarding the concern for compensation, one way to capture this is by means of the following version of the Pigou-Dalton compensation principle, which we call here Pigou-Dalton for Equal Preferences, Efforts and Lifetimes (PDEPEL). This axiom states that, for agents who are identical in terms of everything (i.e. preferences, effort, longevity) except their consumption, a transfer from the agent with the higher consumption to the agent with the lower consumption is a social improvement.

Axiom 4 (Pigou-Dalton for Equal Preferences, Efforts and Lifetimes) For all $R_{N} \in \Re^{|N|}$, all $x_{N}, x_{N}^{\prime} \in X^{|N|}$, and all $i, j \in N$, if $R_{i}=R_{j}$, if $e_{i}=e_{i}^{\prime}=e_{j}=e_{j}^{\prime}, \lambda\left(x_{i}\right)=\lambda\left(x_{i}^{\prime}\right)=\lambda\left(x_{j}\right)=\lambda\left(x_{j}^{\prime}\right)=\ell$, and there exists $\delta \in \mathbb{R}_{++}^{\ell}$ such that

$$
c_{i}^{\prime} \gg c_{i}=c_{i}^{\prime}-\delta \gg c_{j}=c_{j}^{\prime}+\delta \gg c_{j}^{\prime}
$$

\footnotetext{
${ }^{6}$ At the stage of defining the social objective, it is important to posit basic principles like "saving one life is worth some effort" that bear on the evaluation of final consequences. At the stage of policy decisions, studied in later sections, one deals with statistical lives.
} 
and $x_{k}=x_{k}^{\prime}$ for all $k \neq i, j$, then

$$
x_{N} \succsim_{R_{N}} x_{N}^{\prime}
$$

Although quite intuitive, the Pigou-Dalton principle is limited to individuals with identical preferences and this is a very strong limitation. Social orderings that give full priority to individuals having certain kinds of preferences, even when they are extremely better off than others, can satisfy this axiom.

In order to introduce some inequality aversion across individuals with different preferences, a natural candidate consists of the following axiom, which is another version of the Pigou-Dalton principle. This axiom, called the PigouDalton Principle for Constant Consumption, and Reference Effort and Lifetime, states that, if two agents have a longevity that is equal to a level of reference $\ell^{*}$, as well as an effort profile that is equal to a reference profile $e^{*}$, then a transfer that lowers the constant consumption profile of the rich and raises the constant consumption profile of the poor constitutes a social improvement, whatever their preferences.

Axiom 5 (Pigou-Dalton for Constant Consumption, Reference Effort and Lifetime) For all $R_{N} \in \Re^{|N|}$, all $x_{N}, x_{N}^{\prime} \in X^{|N|}$, and all $i, j \in N$, such that $e_{i}=e_{i}^{\prime}=$ $e_{j}=e_{j}^{\prime}=e^{*}$, and such that $\lambda\left(x_{i}\right)=\lambda\left(x_{i}^{\prime}\right)=\lambda\left(x_{j}\right)=\lambda\left(x_{j}^{\prime}\right)=\ell^{*}$, and $x_{i}$ and $x_{j}$ are constant consumption profiles, if there exists $\delta \in \mathbb{R}_{++}^{\ell^{*}}$ such that

$$
c_{i}^{\prime} \gg c_{i}=c_{i}^{\prime}-\delta \gg c_{j}=c_{j}^{\prime}+\delta \gg c_{j}^{\prime}
$$

and $x_{k}=x_{k}^{\prime}$ for all $k \neq i, j$, then

$$
x_{N} \succsim_{R_{N}} x_{N}^{\prime}
$$

The restriction to specific situations (constant consumption, reference effort and longevity) is necessary for this axiom to remain compatible with Weak Pareto, as is well known in social choice theory. It is intuitive that if one wants to respect individual preferences, it is impossible to make interpersonal comparisons in physical terms in all cases. This is the reason for this restriction, which is not needed, by contrast, when the transfer is made between individuals with identical preferences, and this explains the difference between the two Pigou-Dalton axioms introduced here.

The reference profile of health-improving efforts $e^{*}$ and the reference longevity $\ell^{*}$ can be interpreted as follows. The reference effort profile $e^{*}$ and reference longevity $\ell^{*}$ are such that an external observer could, when comparing the lives of two persons with the effort profile $e^{*}$ and the length $\ell^{*}$, say who is better off than the other by just looking at the constant consumption profiles of those agents, without knowing anything about their preferences. Thus the reference levels of longevity and efforts allow the comparison of agents' well-being directly from their consumption, without caring for their preferences. Naturally, the reference efforts profile $e^{*}$ and reference longevity $\ell^{*}$ are ethical parameters, whose selection can, in combination with the Weak Pareto axiom, have important redistributive consequences. 
The five axioms defined above seem quite appealing, and one would like social preferences to satisfy all of them. However, as stated in the following proposition, the first four axioms are logically incompatible.

Theorem 1 There exists no social ordering function $\succsim$ defined on $\Re^{|N|}$ satisfying Weak Pareto, Hansson Independence, PDEPEL, and Survivors Numbers Count.

The proof of this result is simple and we only provide a sketch of the argument. By a direct adaptation of arguments developed in Fleurbaey et al. (2011), one shows that Weak Pareto, Hansson Independence, and PDEPEL imply an absolute priority for the worse-off individuals. That is, if two persons $i$ and $j$ have the same preferences, but $i$ lies on a higher indifference curve than $j$, pushing $i$ on a lower indifference curve and $j$ on a higher one is socially desirable, whatever the relative size of the shift for the two agents.

However, absolute priority for the worse-off conflicts with the Survivors Numbers Count axiom under general assumptions on individual preferences. To see this, let us first remind that this latter axiom states that, as soon as this guarantees the rise in the longevity of one person (and no change in others' longevities), a generalized rise in the preventive effort levels in the population is socially desirable. Note, however, that such a generalized rise in prevention can be damageable to the worse-off individuals. Take the case where the worse-off individuals are short-lived persons who do not like health efforts. If some additional preventive effort is imposed to them - without increasing their longevity - those persons are made worse off than before, and so rising the overall prevention level in the name of the rise in one person's longevity contradicts the priority given to the worse-offs. Hence some choice is to be made between the Pigou-Dalton axiom and the Survivors Numbers Count axiom.

Before drawing the corollaries from that result, it should be noticed that if some restrictions were imposed on individual preferences, the above impossibility would no longer prevail. If, for instance, individual preferences are monotonic not only in consumption, but also in preventive effort, then imposing a general rise in preventive effort will not worsen the situation of the worse-off individuals, and, thus, will not contradict our absolute priority to the worse-offs.

Having stressed this, it remains that there is no obvious reason why one should impose the monotonicity of individual preferences with respect to effort. Hence, the above impossibility highlights a true dilemma: social preferences cannot exhibit both a concern for the number of survivors and a priority to the worse off. One cannot care both about the prevention against an early death, and about the compensation for an early death. A choice must be made between those two concerns, which appear equally reasonable. The rest of the paper explores two distinct approaches to that dilemma.

\section{Two approaches}

We will explore here two distinct branches made salient by the above dilemma. 
On the one hand, a first approach consists of giving up the concern for prevention against a premature death, while keeping the concern for compensating the short-lived. This can been done by relaxing the Survivors Numbers Count axiom, and by characterizing the social preferences in a context where the compensation of the short-lived matters.

On the other hand, one can keep the concern for a wide prevention against an early death, while giving less priority to the concern for compensating the short-lived. This can be done by keeping the Survivors Numbers Count axiom, while adopting a modified version of the Pigou-Dalton principle.

The rest of this section will explore these two solutions.

\subsection{Priority to compensation}

Let us now explore the first approach, which does not require social preferences to satisfy the Survivors Numbers Count axiom. The question is whether this implies completely giving up any concern for prevention.

The combination of the remaining four axioms suffices to provide a partial but relatively precise characterization of social preferences. In order to investigate what kind of social preferences satisfy those conditions, let us first define the Constant Consumption Profile Equivalent for Reference Lifetime and Effort (CCPERLE).

Definition 1 (CCPERLE) For any $i \in N$, any $R_{i} \in \Re$ and any $x_{i} \in X$, the Constant Consumption Profile Equivalent for Reference Lifetime and Effort (CCPERLE) of $x_{i}$ is the constant consumption profile $\hat{c}_{i}$ such that $\lambda\left(\hat{c}_{i}, e^{*}\right)=\ell^{*}$ and

$$
x_{i} I_{i}\left(\hat{c}_{i}, e^{*}\right)
$$

where $e^{*}$ is the reference effort profile and $\ell^{*}$ is the reference longevity level.

The CCPERLE can be interpreted as the constant consumption path that would, if combined with a reference effort profile $e^{*}$ and a reference longevity $\ell^{*}$, make the agent indifferent with his current life. As such, the CCPERLE can be regarded as a way to homogenize consumptions across individuals, by converting consumptions under different efforts and longevities into some comparable consumptions. The intuition goes as follows. In the present context, where agents may have unequal efforts and unequal longevities, looking at consumption profiles does not suffice to have an idea of individual well-being. A given consumption per period does not yield the same well-being if it is associated with a high or a low health effort, or if it is enjoyed during a more or less long life. However, the CCPERLE allows us to have a precise view of agent's well-being, since it has, by construction, taken effort and longevity differentials into account. ${ }^{7}$

\footnotetext{
${ }^{7}$ Note that the CCPERLE of $x_{i}$ always exists if $\ell^{*}=T$, by our assumptions made on $\Re$, but the existence of the CCPERLE is not guaranteed if $\ell^{*}<T$. It may happen that $x_{i}$ with high longevity is strictly preferred to all lifetime consumption profiles with lower longevity $\ell^{*}$. When this happens, we adopt the convention that the CCPERLE is infinite. This problem
} 
It is straightforward to see that, if $c_{i}$ is a constant consumption profile with $\lambda\left(c_{i}, e^{*}\right)=\ell^{*}$, the CCPERLE is equal to the consumption profile, i.e. $\hat{c}_{i}=c_{i}$. However, if $c_{i}$ is a constant consumption profile with an effort profile equal to the reference profile (i.e. $e_{i}=e^{*}$ ) but with a longevity $\ell$ lower than the reference longevity $\ell^{*}$, then we have $\hat{c}_{i} \gtrless c_{i}$, depending on whether $c_{i}$ lies above or below the critical level making a longer life with that consumption worth being lived. Moreover, if $c_{i}$ is a constant consumption profile with $\ell=\ell^{*}$ but with an effort profile lower than the reference profile (i.e. $e_{i} \ll e^{*}$ ), then we have $\hat{c}_{i} \lessgtr c_{i}$, depending on whether the agent likes or dislikes making healthimproving efforts. If the agent regards effort as desirable, imposing the higher reference effort profile to him would require, to bring indifference with his initial situation, a lower consumption profile, yielding $\hat{c}_{i}<c_{i}$. On the contrary, if the agent dislikes making effort, imposing a higher effort level $e^{*} \gg e_{i}$ will require to raise his consumption profile, in such a way as to maintain indifference with the situation with a lower effort. Then, in that case, we will have $\hat{c}_{i}>c_{i}$. Therefore, when the reference effort profile is higher than the actual effort level, our equivalent is lower for the agents with a taste for effort, and higher for agents who dislike efforts, ceteris paribus.

Having defined the CCPERLE, we can now present the following theorem, which characterizes the social preferences, or, more precisely, states that the Maximin on CCPERLE is a necessary condition for social optimality.

Theorem 2 Assume that the social ordering function $\succsim$ satisfies axioms Weak Pareto, Hansson Independence, PDEPEL and PDCCREL on $\Re^{|N|}$. Then $\succsim$ is such that for all $R_{N} \in \Re^{|N|}$, all $x_{N}, x_{N}^{\prime} \in X^{|N|}$,

$$
\min _{i \in N}\left(\hat{c}_{i}\right)>\min _{i \in N}\left(\hat{c}_{i}^{\prime}\right) \Longrightarrow x_{N} \succ_{R_{N}} x_{N}^{\prime} .
$$

This theorem is an immediate extension of Th. 1 in Fleurbaey et al. (2010). As explained in the previous section, the combination of Weak Pareto, Hansson Independence and PDEPEL implies absolute priority for the worse-off among agents having the same preferences. Adding PDCCREL to the list forces interpersonal comparisons to be made in terms of CCPERLE.

It should be noted that this theorem does not give a full characterization of social preferences because it does not say how to compare allocations for which $\min \left(\hat{c}_{i}\right)=\min \left(\hat{c}_{i}^{\prime}\right){ }^{8}$ All the theorem states is that if one allocation exhibits a higher minimum CCPERLE than another, then it must also be socially more desirable. In other words, the theorem implies that maximizing $\min \left(\hat{c}_{i}\right)$ is a necessary operation, as the best social allocation is necessarily included in the set of allocations that maximize $\min \left(\hat{c}_{i}\right)$. Note, however, that in most concrete problems, it is likely that the Maximin on CCPERLE has, as a solution, a

of non-existence is not very important as the social preferences highlighted here focus on the worst-off individuals.

${ }^{8}$ Clearly, given the postulated axioms, the equality of the $\min \left(\hat{c}_{i}\right)$ for two allocations does not necessarily imply social indifference between these allocations: an allocation could still be regarded as better than the other (on the grounds of other aspects of the distribution), and the theorem has nothing to say about that. 
unique allocation, in which case that allocation must also be the most socially desirable allocation. When a unique solution is not obtained, it is natural to refine the Maximin into the Leximin, which extends the lexicographic priority of the worse-off to higher ranks in the distribution.

\subsection{Priority to prevention}

The alternative approach consists in dropping the first Pigou-Dalton axiom and reintroducing Survivors Numbers Count. There are many social ordering functions that satisfy the list of axioms made of Weak Pareto, Hansson Independence, Survivors Numbers Count and PDCCREL. Some of them violate the principle of anonymity, or give different degrees of priority to various individuals as a function of properties of their indifference sets.

However, there is a salient family in the class of social ordering functions satisfying these axioms. Consider two additional and standard axioms.

Axiom 6 (Continuity) For all $R_{N} \in \Re^{|N|}$, all $x_{N} \in X^{|N|}$, the sets

$$
\left\{x_{N}^{\prime} \in X^{|N|} \mid x_{N} \succsim_{R_{N}} x_{N}^{\prime}\right\} \text { and }\left\{x_{N}^{\prime} \in X^{|N|} \mid x_{N}^{\prime} \succsim_{R_{N}} x_{N}\right\}
$$

are closed.

Axiom 7 (Separability) For all $R_{N} \in \Re^{|N|}$, all $x_{N}, x_{N}^{\prime} \in X^{|N|}$, all $M \subseteq N$,

$$
\left(x_{M}, x_{N \backslash M}\right) \succsim_{R_{N}}\left(x_{M}^{\prime}, x_{N \backslash M}\right) \Leftrightarrow\left(x_{M}, x_{N \backslash M}^{\prime}\right) \succsim_{R_{N}}\left(x_{M}^{\prime}, x_{N \backslash M}^{\prime}\right) .
$$

One then obtains the following result, characterizing what we will call the Sum of Transformed CCPERLE.

Theorem 3 Assume that the social ordering function $\succsim$ satisfies Weak Pareto, Hansson Independence, Continuity, Separability, and PDCCREL on $\Re^{|N|}$. Then Survivors Numbers Count is also satisfied, and there is an increasing concave function $\varphi: \mathbb{R}_{+} \rightarrow \mathbb{R}$ such that for all $R_{N} \in \Re^{|N|}$, all $x_{N}, x_{N}^{\prime} \in X^{|N|}$,

$$
x_{N} \succsim_{R_{N}} x_{N}^{\prime} \Leftrightarrow \sum_{i \in N} \varphi\left(\hat{c}_{i}\right) \geq \sum_{i \in N} \varphi\left(\hat{c}_{i}^{\prime}\right) .
$$

Proof. See the Appendix.

Note that this second approach retains a concern for prevention without completely dropping the ideal of compensation. Seeking equality in terms of CCPERLE implies giving some priority to the worse-off. It may happen that for individuals who are far from the reference longevity and effort, and for particular preferences, the social ordering fails to give priority to the distribution of consumption $c$ to the worse-off as measured by CCPERLE. But the ordering never fails to give priority to the worse-off who are at the reference levels, and the more inequality averse the function $W$, the more this priority extends to situation away from the reference levels. 


\section{First-best optimum}

The previous section explored two approaches to the dilemma between prevention against early death and compensation for early death. We showed that, if priority is given to compensation, basic axioms on social preferences imply that the optimal allocation must maximize the minimum CCPERLE in the population. On the contrary, if the survivors number matters, then, provided we add another version of the Pigou-Dalton principle, a wide class of social objective can satisfy our ethical requirements, including social welfare functions that are continuous and quasi-concave in individual CCPERLE levels, and allows for various arbitrages between compensation and prevention concerns.

This section characterizes, under these two alternative social objectives, the social optimum in a resource allocation problem where individuals, who face risky lifetime, differ in time preferences and in (dis)utility from prevention.

\subsection{Environment}

Consider an economy where agents live either one or two periods. The length of life of each agent is only known ex post. Ex ante, the social planner knows individual preferences, as well as the statistical distribution of individual longevities for each level of effort. ${ }^{9}$ The social planner, who can monitor individual effort, looks for the optimum allocation of an endowment $W$ of resources.

Heterogeneity concerns two aspects of preferences: time preferences $\beta_{i}$ and attitude towards effort $v_{j}$. Some agents assign a high weight to the future (old age), whereas others are less patient, and assign a low weight to the future. Some agents like prevention effort (e.g. jogging), whereas others dislike it.

For simplicity, individual lifetime welfare takes a standard time-additive form, which is separable in the utility or disutility of effort:

$$
\begin{aligned}
U_{i j}^{1} & =u\left(c_{i j}\right)+v_{j}\left(e_{i j}\right), \\
U_{i j}^{2} & =u\left(c_{i j}\right)+v_{j}\left(e_{i j}\right)+\beta_{i} u\left(d_{i j}\right),
\end{aligned}
$$

where $c_{i j}, d_{i j}$ and $e_{i j}$ denote first- and second-period consumptions and effort of an agent with a time preference factor $\beta_{i}$ and an utility from effort $v_{j}$, while $U_{i j}^{1}$ and $U_{i j}^{2}$ denote his actual lifetime utility if he lives respectively one or two periods. Temporal utility $u(\cdot)$ takes the same form for everyone, unlike the utility from effort, which is type-specific, under the form of $v_{j}(\cdot) .{ }^{10}$ We assume that there exists some consumption $\bar{c}$ such that $u(\bar{c})=0 .{ }^{11}$

Ex ante, agents are standard expected-utility maximizers:

$$
E U_{i j}=u\left(c_{i j}\right)+v_{j}\left(e_{i j}\right)+\pi\left(e_{i j}\right) \beta_{i} u\left(d_{i j}\right)
$$

where $E U_{i j}$ denotes the expected utility of an agent with time preference $\beta_{i}$ and attitude towards effort $v_{j}\left(e_{i j}\right)$. The expression $\pi\left(e_{i j}\right)$ denotes the survival

\footnotetext{
${ }^{9}$ The latter piece of information amounts, in the present context, to know how the individual effort level affects the life expectancy of agents.

${ }^{10}$ As usual, we assume: $u^{\prime}\left(c_{i j}\right)>0$ and $u^{\prime \prime}\left(c_{i j}\right)<0$.

${ }^{11}$ This amount to assume that $u(0)<0$, which is standard (see Becker et al. 2005).
} 
function for an agent making an effort $e_{i j}$. As usual, we assume $\pi(0)=\tilde{\pi}>0$, $\pi^{\prime}(\cdot)>0, \pi^{\prime \prime}(\cdot)<0$ and that there exists a maximum effort level $\bar{e}$, so that $\pi(\bar{e})=\bar{\pi}<1$.

Heterogeneity takes the following form. Ex ante, agents differ in their time preferences, $\beta_{i}$, and in their attitude towards effort, $v_{j}$, with two types for each. For time preferences, type- $1 j$ agents are less patient than type- $2 j$ agents:

$$
0<\beta_{1}<\beta_{2}<1
$$

Regarding effort, we assume that type- $i 1$ agents dislike efforts, whereas type$i 2$ agents like effort. For simplicity, we assume that $v_{j}(\cdot)=\gamma_{j} v(\cdot)$ with $v(0)=$ $0, v(\cdot) \geq 0, v^{\prime}(\cdot)>0$ and $v^{\prime \prime}(\cdot)>0$, with:

$$
\gamma_{1}<0<\gamma_{2}
$$

Hence, there exist 4 types of agents ex ante, who are differentiated by their $\beta_{i}$ and $\gamma_{j}$. Ex post, there are 8 types of agents, as each ex ante type includes short-lived and long-lived agents. ${ }^{12}$

We assume that the social planner can allocate resources as first-period or second-period consumptions without any cost, but that agents cannot transfer resources across periods freely, so that the bundles (received from the planner) have to be consumed in the same periods as they are received. ${ }^{13}$ Within that framework, the problem of the social planner consists in offering four consumption and effort bundles $\left(c_{i j}, d_{i j}, e_{i j}\right)$ to agents with time preference parameter $\beta_{i}$ and attitude towards efforts $\gamma_{j}$, for $i=1,2$ and $j=1,2$. Note that these bundles do not depend on whether agents live one or two periods, as the actual length of life is not known ex ante by the planner.

The two social objectives considered here are the Maximin CCPERLE and the Sum of Transformed CCPERLE. Note that, at first glance, one might want to consider simpler social objective functions, defined not in terms of CCPERLE levels, but, rather, in terms of individual lifetime welfare levels. The problem is that such an approach would presuppose a full comparability of individual lifetime welfare levels, which is problematic, since preference parameters $\beta_{i}$ and $\gamma_{j}$ then become, under that approach, scaling factors affecting the social optimum in an arbitrary way. ${ }^{14}$ Social objectives defined in terms of CCPERLE levels are immunized against those comparability / aggregation problems.

\subsection{Maximin CCPERLE}

In the following, we solve the problem faced by a planner trying to maximize the minimum CCPERLE, assuming that the planner can observe characteristics $\beta_{i}$ and $\gamma_{j}$. For that purpose, we take the maximum length $\ell=2$ as a reference level $\ell^{*}$, and the maximum effort level $\bar{e}$ as the reference level of effort $e^{*}$.

\footnotetext{
${ }^{12}$ We assume that there is a mass 1 of individuals in each of the ex ante groups.

${ }^{13}$ This amounts to assume that the social planner can fully tax the savings of individuals.

${ }^{14}$ There is, for instance, no obvious reason why, under zero effort, effort-lover and effortaverse individuals should be equally well-off. Moreover, there is no obvious reason why impatient agents should have a lower lifetime welfare than patient agents ceteris paribus.
} 
By definition, the CCPERLE for an agent of type $\left(\beta_{i}, \gamma_{j}\right)$ with an actual length of life $\ell=1,2$ is the constant consumption profile $\left(\hat{c}_{i j \ell}, \hat{c}_{i j \ell}\right)$ such that:

$$
\begin{aligned}
& u\left(\hat{c}_{i j 2}\right)+\gamma_{j} v(\bar{e})+\beta_{i} u\left(\hat{c}_{i j 2}\right)=u\left(c_{i j}\right)+\gamma_{j} v\left(e_{i j}\right)+\beta_{i} u\left(d_{i j}\right) \\
& u\left(\hat{c}_{i j 1}\right)+\gamma_{j} v(\bar{e})+\beta_{i} u\left(\hat{c}_{i j 1}\right)=u\left(c_{i j}\right)+\gamma_{j} v\left(e_{i j}\right)
\end{aligned}
$$

On the first line, $\hat{c}_{i j 2}$ defines the consumption equivalent of an agent with time preference $\beta_{i}$ and attitude towards effort $\gamma_{j}$ who effectively lived two periods, while the second line defines a consumption equivalent $\hat{c}_{i j 1}$ for a $\left(\beta_{i}, \gamma_{j}\right)$-type agent living only one period. Note that since we take ex-post utilities on the right-hand side of these expressions, the CCPERLE of an agent does not depend on his survival probability. However, the ex post lifetime welfare does depend on individual health-affecting effort, through its impact on first-period welfare. Thus, if individual effort depends on the survival function, this will affect indirectly the level of the consumption equivalent for a given longevity.

Let us first define the consumption equivalent $\left(\hat{c}_{i j \ell}, \hat{c}_{i j \ell}\right)$ for each of the 8 groups of individuals that emerge ex post.

\begin{tabular}{ccc|c}
$\beta$ & $\gamma$ & $\ell$ & def. CCPERLE $\hat{c}_{i j \ell}$ \\
\hline$\beta_{1}$ & $\gamma_{1}$ & 1 & $u\left(\hat{c}_{111}\right)\left(1+\beta_{1}\right)+\gamma_{1} v(\bar{e})=u\left(c_{11}\right)+\gamma_{1} v\left(e_{11}\right)$ \\
$\beta_{1}$ & $\gamma_{2}$ & 1 & $u\left(\hat{c}_{121}\right)\left(1+\beta_{1}\right)+\gamma_{2} v(\bar{e})=u\left(c_{12}\right)+\gamma_{2} v\left(e_{12}\right)$ \\
$\beta_{2}$ & $\gamma_{1}$ & 1 & $u\left(\hat{c}_{211}\right)\left(1+\beta_{2}\right)+\gamma_{1} v(\bar{e})=u\left(c_{21}\right)+\gamma_{1} v\left(e_{21}\right)$ \\
$\beta_{2}$ & $\gamma_{2}$ & 1 & $u\left(\hat{c}_{221}\right)\left(1+\beta_{2}\right)+\gamma_{2} v(\bar{e})=u\left(c_{22}\right)+\gamma_{2} v\left(e_{22}\right)$ \\
$\beta_{1}$ & $\gamma_{1}$ & 2 & $u\left(\hat{c}_{112}\right)\left(1+\beta_{1}\right)+\gamma_{1} v(\bar{e})=u\left(c_{11}\right)+\gamma_{1} v\left(e_{11}\right)+\beta_{1} u\left(d_{11}\right)$ \\
$\beta_{1}$ & $\gamma_{2}$ & 2 & $u\left(\hat{c}_{122}\right)\left(1+\beta_{1}\right)+\gamma_{2} v(\bar{e})=u\left(c_{12}\right)+\gamma_{2} v\left(e_{12}\right)+\beta_{1} u\left(d_{12}\right)$ \\
$\beta_{2}$ & $\gamma_{1}$ & 2 & $u\left(\hat{c}_{212}\right)\left(1+\beta_{2}\right)+\gamma_{1} v(\bar{e})=u\left(c_{21}\right)+\gamma_{1} v\left(e_{21}\right)+\beta_{2} u\left(d_{21}\right)$ \\
$\beta_{2}$ & $\gamma_{2}$ & 2 & $u\left(\hat{c}_{222}\right)\left(1+\beta_{2}\right)+\gamma_{2} v(\bar{e})=u\left(c_{22}\right)+\gamma_{2} v\left(e_{22}\right)+\beta_{2} u\left(d_{22}\right)$
\end{tabular}

The problem of the social planner can thus be written as follows:

$$
\begin{aligned}
& \max _{c_{i j}, d_{i j}, e_{i j}} \min \left(\hat{c}_{111}, \hat{c}_{121}, \hat{c}_{211}, \hat{c}_{221}, \hat{c}_{112}, \hat{c}_{122}, \hat{c}_{212}, \hat{c}_{222}\right) \\
& \text { s.to } c_{11}+\pi\left(e_{11}\right) d_{11}+c_{21}+\pi\left(e_{21}\right) d_{21}+c_{12}+\pi\left(e_{12}\right) d_{12}+c_{22}+\pi\left(e_{22}\right) d_{22} \leq W
\end{aligned}
$$

where the CCPERLE $\hat{c}_{i j \ell}$ are defined in the above table.

The solution to that social planning problem is represented below.

Proposition 1 Under perfect information about ex-ante types $\left(\beta_{i}, \gamma_{j}\right)$, and $(4+2 \tilde{\pi}+2 \bar{\pi}) \bar{c} \leq W$, the Maximin CCPERLE optimum involves:

$$
\begin{aligned}
\bar{c} & <c_{11}^{*}<c_{12}^{*}, c_{21}^{*}<c_{22}^{*} \\
d_{11}^{*} & =d_{12}^{*}=d_{21}^{*}=d_{22}^{*}=\bar{c} \\
e_{11}^{*} & =e_{21}^{*}=0<e_{12}^{*}=e_{22}^{*}=\bar{e}
\end{aligned}
$$

That consumption, effort profile leads to an equalization of CCPERLE among ex ante groups, but may not prevent inequalities within ex ante groups:

$$
\begin{aligned}
& \hat{c}_{121}^{*}=\hat{c}_{122}^{*} \leq \hat{c}_{111}^{*}=\hat{c}_{112}^{*} \quad \text { and } \quad \hat{c}_{221}^{*}=\hat{c}_{222}^{*} \leq \hat{c}_{211}^{*}=\hat{c}_{212}^{*} \\
& \hat{c}_{211}^{*}=\hat{c}_{212}^{*} \leq \hat{c}_{111}^{*}=\hat{c}_{112}^{*} \quad \text { and } \quad \hat{c}_{221}^{*}=\hat{c}_{222}^{*} \leq \hat{c}_{121}^{*}=\hat{c}_{122}^{*}
\end{aligned}
$$


Proof. See the Appendix.

The Maximin CCPERLE optimum exhibits three major features.

First, it provides a consumption $\bar{c}$ to all agents at the old age. Indeed, if $d_{i j}$ exceeds $\bar{c}$, long-lived agents are better off than short-lived agents. Hence, redistributing $d_{i j}-\bar{c}$ towards the young increases the welfare of the short-lived. Inversely, if $d_{i j}<\bar{c}$, the worst-off agents are the long-lived, so that redistributing towards them until $d_{i j}=\bar{c}$ would raise the welfare of the worst-offs. Hence we must have $d_{i j}=\bar{c}^{15}$

Second, the Maximin CCPERLE imposes zero prevention effort on agents who dislike prevention. The reason is that, since some effort-averse agents will turn out to be short-lived despite prevention, the welfare of those short-lived agents is definitely increased by allowing them to avoid prevention effort. The same is not true for effort-loving agents, for whom it is always optimal to allow them to do the maximum effort, whatever they survive to the old age or not.

Third, the Maximin CCPERLE discriminates, at the young age, between agents with different ex ante characteristics. The priority given to patient agents (for a given attitude towards prevention) is not surprising, since patient agents are more affected by a short life than impatient agents. Regarding the priority given to those who like prevention, the intuition goes as follows. Agents who dislike effort tend to choose an effort level that is inferior to the reference effort, and this suffices to make these appear "better off" than the agents who like efforts and practice the reference effort. Hence, when one measures the wellbeing of agents by using the maximum effort level as a reference, the CCPERLE of the patient who likes effort is necessarily lower, for an equal first-period consumption, than the CCPERLE of the patient who dislikes effort. Hence the Maximin CCPERLE leads to higher first-period consumption for agents who like prevention (under given time preferences).

All in all, the Maximin CCPERLE achieves a full compensation with respect to the length of life: for given $\beta_{i}$ and $\gamma_{j}$, there is, at the social optimum, an equality of lifetime welfare, whatever individuals live long or not. However, there may remain some welfare inequalities across agents with an equal longevity, but these inequalities result from agents' preferences (different $\beta_{i}$ or $\gamma_{j}$ ), for which they can be held responsible, unlike for a premature death. ${ }^{16}$

Regarding the prevention, imposing zero effort levels to all agents who do not life effort may be shocking. Indeed, given that the proportion of survivors in a group depends on the amount of effort, imposing such a low effort level amounts to nothing less than reducing the number of survivors with respect to what would have prevailed under, for instance, a generalized high effort. But the Maximin solution does not depend on the number of agents who are in each situation (e.g. short-lived or long-lived), but only on how bad the situation of the worse-off is. This explains why it is optimal to impose effort levels that are minimal for agents who do not like effort. Allowing large efforts for those agents

\footnotetext{
${ }^{15}$ That rationale is close to the one used in Fleurbaey et al (2011).

${ }^{16} \mathrm{On}$ the distinction between "circonstances" characteristics and "responsibility" characteristics, see Fleurbaey (2008).
} 
would make the short-lived members even worse-off than without such effort. ${ }^{17}$

That particular corollary of the Maximin CCPERLE is counterintuitive, since there is a strong intuitive support for large preventive campaigns favouring large levels of health-improving efforts, and leading, in fine, to a large number of survivors. Obviously the Maximin CCPERLE, which does not care about the number of survivors, but only about the situation of the short-lived, does not legitimate such practices. The reason is that the Maximin CCPERLE gives absolute priority to compensation concerns over prevention concerns.

\subsection{Sum of Transformed CCPERLE}

Let us now explore what the social optimum becomes under an alternative approach to the compensation / prevention dilemma, which does not give up a concern for massive prevention. As stated in Theorem 3, adding the Survivors Numbers Count axiom to the Weak Pareto and Hansson Independence axiom, while replacing the Pigou-Dalton Principle for Equal Preferences, Effort and Lifetime (PDEPEL) by the Pigou-Dalton Principle for Constant Consumption and Reference Effort and Lifetime (PDCCREL), implies, under Continuity and Separability, that social preferences can be represented by a sum of transformed CCPERLE $\varphi\left(\hat{c}_{i j \ell}\right)$, with $\varphi^{\prime}(\cdot)>0$ and $\varphi^{\prime \prime}(\cdot)<0$.

For the purpose of this analytical application, we will take, as a social objective, Atkinson's isoelastic social welfare function, defined in terms of individual CCPERLE (instead of individual utilities as usually done): ${ }^{18}$

$$
W\left(\hat{c}_{N}\right)=\sum_{i, j, \ell} n_{i j \ell} \frac{\left(\hat{c}_{i j \ell}\right)^{1-\nu}-1}{1-\nu}
$$

where $\nu$ reflects the sensitivity of social preferences to inequalities in CCPERLE among the population, while $n_{i j \ell}$ is proportion of individuals of type $i j$ with a length of life $\ell .{ }^{19}$ That social objective includes the case where $\nu=0$, in which what is to be maximized is the sum of individuals' CCPERLE. On the contrary, when $\nu$ tends to $+\infty$, the social objective is the Maximin on CCPERLE, which gives absolute priority to the compensation of the dead.

Except in that special case, the isoelastic social objective function $W\left(\hat{c}_{N}\right)$ does some justice to massive prevention. Indeed, instead of focusing only on the worse-off individuals, it ranks allocations by comparing aggregate outcomes on the population as a whole. But despite that difference, the informational basis relevant for the ranking of allocations still remains individuals' CCPERLE,

\footnotetext{
${ }^{17}$ The same conclusion remains true when the long-lived are the worst-offs. Note also that, in that case, it is still optimal to allow effort-lovers to do as much effort as possible, since effort-lovers enjoy the effort whatever they will survive or not to the old age. Thus there is no argument for preventing them from making such efforts.

${ }^{18}$ See Atkinson and Stiglitz (1980, p. 340).

${ }^{19} \mathrm{By}$ assumption, we have $n_{i j}=1$ for all $i, j$, so that $n_{i j 1}=\left(1-\pi_{j}\right) n_{i j}=1-\pi_{j}$, while $n_{i j 2}=\pi_{j} n_{i j}=\pi_{j}$.
} 
which, under reference levels $\ell^{*}=2$ and $e^{*}=\bar{e}$, is:

$$
\hat{c}_{i j \ell}=f\left(\frac{U_{i j}^{\ell}-\gamma_{j} v(\bar{e})}{1+\beta_{i}}\right)
$$

where $f(x) \equiv u^{-1}(x)$. As $u^{\prime}(x)>0$ and $u^{\prime \prime}(x)<0$, we have $f^{\prime}(x)>0$ and $f^{\prime \prime}(x)>0$ : the CCPERLE is an increasing convex function. Using the notations $g\left(\hat{c}_{i j \ell}\right) \equiv \frac{\left(\hat{c}_{i j \ell}\right)^{1-\nu}-1}{1-\nu}$, the transformed CCPERLE can be rewritten as:

$$
g\left(\hat{c}_{i j \ell}\right)=g \circ f\left(\frac{U_{i j}^{\ell}-\gamma_{j} v(\bar{e})}{1+\beta_{i}}\right) \equiv h\left(\frac{U_{i j}^{\ell}-\gamma_{j} v(\bar{e})}{1+\beta_{i}}\right)
$$

where $h(x) \equiv g \circ f(x)$. Moreover, if one denotes $f\left(\frac{U_{i j}^{1}-\gamma_{j} v(\bar{e})}{1+\beta_{i}}\right) \equiv f_{i j 1}\left(c_{i j}, e_{i j}\right)$ and $f\left(\frac{U_{i j}^{2}-\gamma_{j} v(\bar{e})}{1+\beta_{i}}\right) \equiv f_{i j 2}\left(c_{i j}, d_{i j}, e_{i j}\right)$, we can define $h_{i j 1}\left(c_{i j}, e_{i j}\right) \equiv g \circ f_{i j 1}\left(c_{i j}, e_{i j}\right)$ and $h_{i j 2}\left(c_{i j}, d_{i j}, e_{i j}\right) \equiv g \circ f_{i j 2}\left(c_{i j}, d_{i j}, e_{i j}\right)$. Hence we have:

$$
\begin{aligned}
& \frac{\partial h_{i j 1}\left(c_{i j}, e_{i j}\right)}{\partial c_{i j}}>0 \text { and } \frac{\partial h_{i j 1}\left(c_{i j}, e_{i j}\right)}{\partial e_{i j}}>0 \text { for } j=1 \\
& \frac{\partial h_{i j 2}\left(c_{i j}, d_{i j}, e_{i j}\right)}{\partial c_{i j}}>0, \frac{\partial h_{i j 2}\left(c_{i j}, d_{i j}, e_{i j}\right)}{\partial d_{i j}}>0 \\
& \begin{aligned}
\frac{\partial h_{i j 2}\left(c_{i j}, d_{i j}, e_{i j}\right)}{\partial e_{i j}} & <0 \text { for } j=1 \\
& >0 \text { for } j=2
\end{aligned}
\end{aligned}
$$

It is not obvious to see whether the composed functions $h_{i j 1}(x)$ and $h_{i j 2}(x)$ are concave or convex in their arguments. In order to guarantee the concavity of the objective function to be maximized, we assume that the transformed CCPERLEs are concave in their arguments (consumptions and prevention). ${ }^{20}$ This assumption is most likely to hold when inequality aversion is large. ${ }^{21}$

The social planner's problem can be rewritten as:

$$
\begin{aligned}
& \max _{c_{i j}, d_{i j}, e_{i j}} \sum_{i, j} n_{i j 1} h_{i j 1}\left(c_{i j}, e_{i j}\right)+\sum_{i, j} n_{i j 2} h_{i j 2}\left(c_{i j}, d_{i j}, e_{i j}\right) \\
& \text { s.to } c_{11}+\pi\left(e_{11}\right) d_{11}+c_{21}+\pi\left(e_{21}\right) d_{21}+c_{12}+\pi\left(e_{12}\right) d_{12}+c_{22}+\pi\left(e_{22}\right) d_{22} \leq W
\end{aligned}
$$

${ }^{20}$ This assumption can be written as:

$$
\begin{aligned}
\frac{\partial^{2} h_{i j 1}\left(c_{i j}, e_{i j}\right)}{\partial c_{i j}^{2}} & <0, \frac{\partial^{2} h_{i j 1}\left(c_{i j}, e_{i j}\right)}{\partial e_{i j}^{2}}<0 \\
\frac{\partial^{2} h_{i j 2}\left(c_{i j}, d_{i j}, e_{i j}\right)}{\partial c_{i j}^{2}} & <0, \frac{\partial^{2} h_{i j 2}\left(c_{i j}, d_{i j}, e_{i j}\right)}{\partial d_{i j}^{2}}<0, \frac{\partial^{2} h_{i j 2}\left(c_{i j}, d_{i j}, e_{i j}\right)}{\partial e_{i j}^{2}}<0
\end{aligned}
$$

\footnotetext{
${ }^{21}$ However, it is shown in the Appendix, by means of a simple analytical example, that this assumption is actually quite weak, since it is, in general, satisfied even when the degree of inequality aversion is zero (i.e. $\nu=0$ ).
} 
Proposition 2 Under perfect information about ex-ante types $\left(\beta_{i}, \gamma_{j}\right)$, and $(4+4 \bar{\pi}) \bar{c} \leq W$, the Sum of Transformed CCPERLE optimum involves:

$$
\begin{aligned}
& c_{i 1}^{* *}<c_{i 2}^{* *} \forall i \\
& \left\{\begin{array}{c}
c_{1 j}^{* *}<c_{2 j}^{* *} \text { when low } \pi(\cdot) \\
c_{1 j}^{* *}>c_{2 j}^{* *} \text { when high } \pi(\cdot)
\end{array}\right. \\
& c_{i j}^{* *}>d_{i j}^{* *} \forall i, j \\
& d_{22}^{* *}>d_{12}^{* *}, d_{21}^{* *}>d_{11}^{* *}>\bar{c} \\
& e_{12}^{* *}=e_{22}^{* *}=\bar{e}>e_{21}^{* *}>e_{11}^{* *}>0
\end{aligned}
$$

Proof. See the Appendix.

Thus our alternative social optimum involves higher first-period consumption for agents who like effort than for those who dislike effort, since their CCPERLE exhibits a higher marginal rise when first-period consumption increases. Moreover, whether patient agents receive more or less consumption in the first period that impatient agents depends on the level of survival probabilities. If survival chances are low (whatever the effort is), the most relevant group consists of short-lived individuals. Hence, given that the more reactive CCPERLE among the short-lived are those of patient agents, these should receive higher firstperiod consumption. On the contrary, if survival chances are high (whatever the effort is), the most relevant group consists of survivors. Then, the most reactive CCPERLE is the one of impatient agents, who should then receive higher consumption at the young age.

The social optimum also involves declining consumption profiles. Regarding the levels of second-period consumption, these all exceed $\bar{c}$, and the largest oldage consumption concerns patient agents who like efforts, whereas the lowest old-age consumption is for impatient agents who dislike effort.

Finally, the social optimum involves a positive prevention effort for all individuals, even though agents who like efforts should still, at the social optimum, make more efforts than effort-averse agents.

We are now in position to compare the social optimum under the Sum of Transformed CCPERLE with the one under the Maximin CCPERLE.

Corollary 1 Comparing the social optima under Maximin CCPERLE (*) and under the Sum of Transformed CCPERLE (**), we have:

$$
\begin{aligned}
& c_{1 j}^{*}<c_{2 j}^{*} \text { but }\left\{\begin{array}{c}
c_{1 j}^{* *}<c_{2 j}^{* *} \text { when low } \pi(\cdot) \\
c_{1 j}^{* *}>c_{2 j}^{* *} \text { when high } \pi(\cdot)
\end{array}\right. \\
& c_{i 1}^{*}<c_{i 2}^{*} \text { and } c_{i 1}^{* *}<c_{i 2}^{* *} \\
& d_{11}^{*}=d_{12}^{*}=d_{21}^{*}=d_{22}^{*}<d_{11}^{* *}<d_{12}^{* *}, d_{21}^{* *}<d_{22}^{* *} \\
& e_{11}^{*}=e_{21}^{*}=0<e_{11}^{* *}=e_{21}^{* *} \\
& e_{12}^{*}=e_{22}^{*}=\bar{e}=e_{12}^{* *}=e_{22}^{* *}
\end{aligned}
$$

Proof. The proof follows from Propositions 1 and 2 . 
There are three major differences between those alternative social optima.

First, in terms of prevention, whereas the Maximin CCPERLE recommends no preventive effort for agents who dislike these, on the grounds of the welfare of those who turn out to be short-lived despite efforts, the Sum of Transformed CCPERLE legitimates prevention efforts for all, but with, nonetheless, lower efforts for those who dislike efforts. That major difference is due to the fact that the latter social objective, takes - unlike the former - the number of survivors into account, and not only the welfare of the short-lived.

Second, whereas the Maximin CCPERLE assigns a low consumption $\bar{c}$ to all surviving old individuals, the Sum of Transformed CCPERLE provides higher old-age consumption to all survivors, while favouring the patient over the impatient, and effort-lover over effort-averse agents. This major departure with respect to the Maximin CCPERLE optimum comes from the aggregated nature of the Sum of Transformed CCPERLE objective, which cares also about the welfare of the old individuals, even though these are not the worst-offs.

Third, there is also some major difference regarding the treatment of heterogeneity at the young age. The Maximin CCPERLE favours patient agents (for whom the welfare loss due to premature death is the largest). On the contrary, the Sum of Transformed CCPERLE favours, for sufficiently large survival chances, impatient agents over patient agents, since, in case of widespread survival to the old age, the CCPERLE of impatient agents is more reactive to $c_{i j}$ than the one of patient agents. Finally, both the Maximin CCPERLE and the Sum of Transformed CCPERLE favour agents who like effort (since effort-averse are supposed to benefit from a lower effort than the reference level, which makes their CCPERLE less reactive to a marginal rise in $c_{i j}$ ).

The Sum of Transformed CCPERLE, by leading to a higher prevention and to a higher level of consumption at the old age, seems less radical than the Maximin CCPERLE. Nonetheless, that social objective faces a major inconvenient: more prevention and more old-age consumption both go against the ideal of compensating short-lived individuals. Thus that alternative social objective does not overcome the dilemma between compensation and prevention. It only provides one approach, which gives up the priority to compensation. Obviously, giving up that priority is not costless.

\section{Extensions}

\subsection{Unobserved ex ante heterogeneity}

Let us now relax the perfect observability assumption on preference parameters $\beta_{i}$ and $\gamma_{j}$, and characterize the second-best social optimum, under the Maximin CCPERLE and the Sum of Transformed CCPERLE.

Focusing on the former social objective, the problem of the social planner is, under that alternative information environment, to propose 4 bundles $\left(c_{i j}, d_{i j}, e_{i j}\right)$, one for each ex ante type, in such a way as to maximize the minimum CCPERLE, still subject to the budget constraint, to which we add incen- 
tive compatibility constraints. Given that, at the first-best, impatient agents may be tempted to pretend to be patient, to receive higher first-period consumption, and that effort-averse agents may also be tempted to pretend to be effort-lover, the incentive compatibility constraints are:

$$
\begin{aligned}
u\left(c_{1 j}\right)+\gamma_{j} v\left(e_{1 j}\right)+\pi\left(e_{1 j}\right) \beta_{1} u\left(d_{1 j}\right) & \geq u\left(c_{2 j}\right)+\gamma_{j} v\left(e_{2 j}\right)+\pi\left(e_{2 j}\right) \beta_{1} u\left(d_{2 j}\right) \quad \forall j=1,2 \\
u\left(c_{i 1}\right)+\gamma_{1} v\left(e_{i 1}\right)+\pi\left(e_{i 1}\right) \beta_{i} u\left(d_{i 1}\right) & \geq u\left(c_{i 2}\right)+\gamma_{1} v\left(e_{i 2}\right)+\pi\left(e_{i 2}\right) \beta_{i} u\left(d_{i 2}\right) \quad \forall i=1,2
\end{aligned}
$$

The solution to that social planning problem is represented below.

Proposition 3 Under imperfect information about ex-ante types $\left(\beta_{i}, \gamma_{j}\right)$, and $(4+2 \tilde{\pi}+2 \bar{\pi}) \bar{c} \leq W$, the Maximin CCPERLE optimum involves:

$$
\begin{aligned}
\bar{c} & <c_{11}^{*}=c_{21}^{*}<c_{12}^{*}=c_{22}^{*} \\
d_{11}^{*} & =d_{12}^{*}=d_{21}^{*}=d_{22}^{*}=\bar{c} \\
e_{11}^{*} & =e_{21}^{*}=0<e_{12}^{*}=e_{22}^{*}=\bar{e}
\end{aligned}
$$

Proof. See the Appendix.

The only difference with respect to the first-best is that patient and impatient agents are here treated equally at the young age, unlike at the first-best, where patient agents received higher first-period consumptions. Note, however, that it remains still true that effort-loving agents receive more first-period consumption than effort-averse agents, exactly as in the first-best. The reason is that, when the social planner offers a bundle with maximum prevention, all effort-averse agents are forced to reveal their true type, to avoid large welfare losses. Thus the first-best inequalities between effort-averse and effort-lover consumption at the young age are, at least qualitatively, conserved at the second-best.

Turning now to the Sum of Transformed CCPERLE, the addition, to the social planner's problem, of incentive compatibility constraints tends to complicate the analysis. However, the extent to which the second-best optimum differs from the first-best optimum can be illustrated numerically. For that purpose, the following table compares the first-best and second-best optima under three degrees of inequality aversion: $\nu=0.5, \nu=2$ and $\nu=5 .^{22}$

First-best figures perfectly illustrate the results in Proposition 2. Effortaverse agents always receive, ceteris paribus, lower consumption than effortloving agents (both at the young and the old age). Moreover, patient agents have higher old-age consumption than impatient agents. However, at the young age, impatient agents receive higher consumption than patient agents when optimal prevention is high, i.e. under $\nu=0.50$, whereas the opposite holds under lower prevention, as under $\nu=2$ and $\nu=5$. Regarding prevention, it is optimal for effort-loving agents to carry out maximum prevention $\bar{e}$, while

\footnotetext{
${ }^{22}$ Here we assume $u\left(c_{i j}\right)=\sqrt[2]{c_{i j}}, v\left(e_{i j}\right)=\frac{e_{i j}^{2}}{2}$, and $\pi\left(e_{i j}\right)=\frac{a+7 e}{b+7 e}$ as well as the following values for our parameters:

\begin{tabular}{cccccccccc}
\hline$\beta_{1}$ & $\beta_{2}$ & $\gamma_{1}$ & $\gamma_{2}$ & $\bar{e}$ & $a$ & $b$ & $W$ & $\ell^{*}$ & $e^{*}$ \\
0.5 & 1.0 & -1 & 1 & 1 & 2 & 3 & 100 & 2 & 1 \\
\hline
\end{tabular}
}


effort-averse agents only do prevention when inequality aversion is sufficiently low.

\begin{tabular}{|c|c|c|c|c|c|c|}
\hline \hline & \multicolumn{2}{|c|}{$\nu=0.5$} & \multicolumn{2}{c|}{$\nu=2.0$} & \multicolumn{2}{c|}{$\nu=5.0$} \\
\hline & $\mathrm{FB}$ & $\mathrm{SB}$ & $\mathrm{FB}$ & $\mathrm{SB}$ & $\mathrm{FB}$ & $\mathrm{SB}$ \\
\hline$c_{11}^{* *}$ & 27.60 & 25.70 & 18.60 & 21.90 & 16.90 & 22.20 \\
$c_{12}^{* *}$ & 29.10 & 27.20 & 23.20 & 25.30 & 21.10 & 26.50 \\
$c_{21}^{* *}$ & 15.40 & 17.10 & 23.60 & 21.30 & 27.10 & 22.20 \\
$c_{22}^{* *}$ & 16.40 & 18.00 & 28.40 & 25.30 & 32.30 & 26.50 \\
\hline$d_{11}^{* *}$ & 2.00 & 1.50 & 1.10 & 2.10 & 0.60 & 1.00 \\
$d_{12}^{* *}$ & 2.00 & 1.50 & 1.10 & 1.70 & 0.60 & 0.70 \\
$d_{21}^{* *}$ & 4.50 & 5.40 & 2.80 & 2.70 & 1.00 & 1.10 \\
$d_{22}^{* *}$ & 4.60 & 5.40 & 2.80 & 1.70 & 1.10 & 0.70 \\
\hline$e_{11}^{* *}$ & 0.52 & 0.51 & 0.00 & 0.00 & 0.00 & 0.02 \\
$e_{12}^{* *}$ & 1.00 & 1.00 & 1.00 & 1.00 & 1.00 & 1.00 \\
$e_{21}^{* *}$ & 0.49 & 0.50 & 0.00 & 0.00 & 0.00 & 0.00 \\
$e_{22}^{* *}$ & 1.00 & 1.00 & 1.00 & 1.00 & 1.00 & 1.00 \\
\hline \hline
\end{tabular}

Turning now to the second-best, the added incentive constraints have the following effects. In order to prevent impatient agents from pretending to be patient, a simple solution consists of proposing consumption bundles with higher second-period consumption for agents who pretend to be patient, in comparison with the first-best. That upward distortion in old-age consumption is observed under low inequality aversion (i.e. $\nu=0.5$ ). However, for higher degrees of inequality aversion, the separation of types is no longer achieved through larger old-age consumption gap between patient and impatient agents, but, rather, through proposing higher first-period consumptions for the impatient, as well as lower first-period consumption for the patient, in comparison to the first-best.

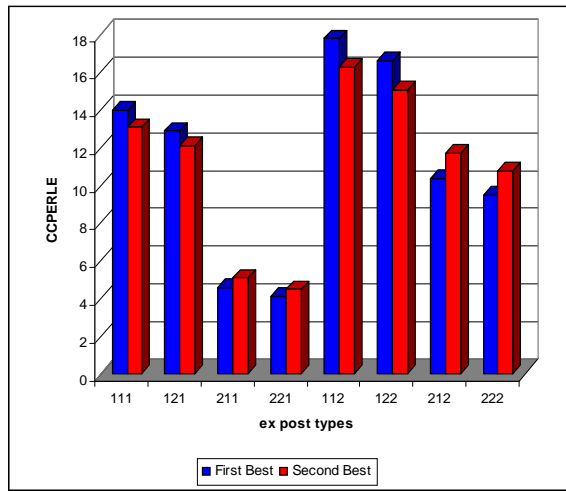

Figure 1: CCPERLE at FB and SB

$$
(\nu=0.5) \text {. }
$$

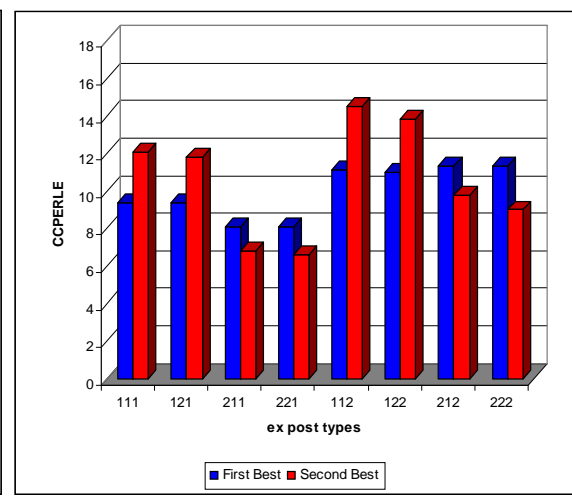

Figure 2: CCPERLE at FB and SB

$$
(\nu=5.00) \text {. }
$$

As far as welfare inequalities are concerned, the comparison of CCPERLE levels shown on Figures 1 and 2 confirms that welfare inequalities between short- 
lived and long-lived agents are significantly reduced when the inequality aversion rises from $\nu=0.5$ (Figure 1) to $\nu=5$ (Figure 2), in the first-best as well as the second-best optimum. Under low inequality aversion (Figure 1), impatient agents are, at the first-best, better off than patient agents, and effort-averse individuals are better off than effort-loving agents ceteris paribus. These inequalities are preserved at the second-best optimum. The only difference is that impatient agents are net "losers" at the second-best (in comparison with the first-best), whereas patient agents are net "winners". In contrast, under high inequality aversion (Figure 2), the first-best involves lower inequalities across ex ante types, for short-lived as well as long-lived agents. Nonetheless, at the second-best, the "winners" - in comparison to the first-best - are now the impatient individuals, and the "losers" are the patient ones. This is an inversion of what is observed under low inequality aversion. Hence, the impact of incentive compatibility constraints in terms of welfare inequalities is sensitive to the degree of inequality aversion.

\subsection{Reference longevity and effort}

Let us now assess the robustness of our results to the reference levels for longevity $\ell$ and prevention $e$. For that purpose, we first take the minimum longevity $\ell=1$ as the reference longevity $\ell^{*}$, while still assuming reference effort $e^{*}=\bar{e}$. The following table shows the CCPERLE for the 8 types of agents ex post.

\begin{tabular}{ccc|c}
$\beta$ & $\gamma$ & $\ell$ & def. CCPERLE $\hat{c}_{i j \ell}$ \\
\hline$\beta_{1}$ & $\gamma_{1}$ & 1 & $u\left(\hat{c}_{111}\right)+\gamma_{1} v(\bar{e})=u\left(c_{11}\right)+\gamma_{1} v\left(e_{11}\right)$ \\
$\beta_{1}$ & $\gamma_{2}$ & 1 & $u\left(\hat{c}_{121}\right)+\gamma_{2} v(\bar{e})=u\left(c_{12}\right)+\gamma_{2} v\left(e_{12}\right)$ \\
$\beta_{2}$ & $\gamma_{1}$ & 1 & $u\left(\hat{c}_{211}\right)+\gamma_{1} v(\bar{e})=u\left(c_{21}\right)+\gamma_{1} v\left(e_{21}\right)$ \\
$\beta_{2}$ & $\gamma_{2}$ & 1 & $u\left(\hat{c}_{221}\right)+\gamma_{2} v(\bar{e})=u\left(c_{22}\right)+\gamma_{2} v\left(e_{22}\right)$ \\
$\beta_{1}$ & $\gamma_{1}$ & 2 & $u\left(\hat{c}_{112}\right)+\gamma_{1} v(\bar{e})=u\left(c_{11}\right)+\gamma_{1} v\left(e_{11}\right)+\beta_{1} u\left(d_{11}\right)$ \\
$\beta_{1}$ & $\gamma_{2}$ & 2 & $u\left(\hat{c}_{122}\right)+\gamma_{2} v(\bar{e})=u\left(c_{12}\right)+\gamma_{2} v\left(e_{12}\right)+\beta_{1} u\left(d_{12}\right)$ \\
$\beta_{2}$ & $\gamma_{1}$ & 2 & $u\left(\hat{c}_{212}\right)+\gamma_{1} v(\bar{e})=u\left(c_{21}\right)+\gamma_{1} v\left(e_{21}\right)+\beta_{2} u\left(d_{21}\right)$ \\
$\beta_{2}$ & $\gamma_{2}$ & 2 & $u\left(\hat{c}_{222}\right)+\gamma_{2} v(\bar{e})=u\left(c_{22}\right)+\gamma_{2} v\left(e_{22}\right)+\beta_{2} u\left(d_{22}\right)$
\end{tabular}

The problem of the social planner can thus be written as follows:

$$
\max _{c_{i j}, d_{i j}, e_{i j}} \min \left(\hat{c}_{111}, \hat{c}_{121}, \hat{c}_{211}, \hat{c}_{221}, \hat{c}_{112}, \hat{c}_{122}, \hat{c}_{212}, \hat{c}_{222}\right) \quad \text { s.t. BC }
$$

where the CCPERLE $\hat{c}_{i j \ell}$ are defined in the above table.

Proposition 4 Under perfect information about ex-ante types $\left(\beta_{i}, \gamma_{j}\right)$, and $(4+2 \tilde{\pi}+2 \bar{\pi}) \bar{c} \leq W$, the Maximin CCPERLE optimum involves, under $\ell^{*}=1$ :

$$
\begin{aligned}
\bar{c} & <c_{11}^{*}=c_{21}^{*}<c_{22}^{*}=c_{12}^{*} \\
d_{11}^{*} & =d_{12}^{*}=d_{21}^{*}=d_{22}^{*}=\bar{c} \\
e_{11}^{*} & =e_{21}^{*}=0<e_{12}^{*}=e_{22}^{*}=\bar{e}
\end{aligned}
$$


Proof. See the Appendix.

The only difference with respect to the Maximin CCPERLE under $\ell^{*}=$ 2 concerns the treatment of patient and impatient agents at the young age. Whereas, under $\ell^{*}=2$, patient agents receive higher consumption at young age, this is no longer true under $\ell^{*}=1$, where they are treated equally, because the short-lived's CCPERLE is independent from time preferences under $\ell^{*}=1$.

Note also that the second-best Maximin CCPERLE, at which patient and impatient agents have the same first-period consumption, is fully robust to the selection of the reference longevity level (unlike the first-best optimum).

Take now the case where the reference effort level is the minimum effort level: $e^{*}=0$. The list of CCPERLE becomes, under $\ell^{*}=2$ :

\begin{tabular}{ccc|c}
$\beta$ & $\gamma$ & $\ell$ & def. CCPERLE $\hat{c}_{i j \ell}$ \\
\hline$\beta_{1}$ & $\gamma_{1}$ & 1 & $u\left(\hat{c}_{111}\right)\left(1+\beta_{1}\right)=u\left(c_{11}\right)+\gamma_{1} v\left(e_{11}\right)$ \\
$\beta_{1}$ & $\gamma_{2}$ & 1 & $u\left(\hat{c}_{121}\right)\left(1+\beta_{1}\right)=u\left(c_{12}\right)+\gamma_{2} v\left(e_{12}\right)$ \\
$\beta_{2}$ & $\gamma_{1}$ & 1 & $u\left(\hat{c}_{211}\right)\left(1+\beta_{2}\right)=u\left(c_{21}\right)+\gamma_{1} v\left(e_{21}\right)$ \\
$\beta_{2}$ & $\gamma_{2}$ & 1 & $u\left(\hat{c}_{221}\right)\left(1+\beta_{2}\right)=u\left(c_{22}\right)+\gamma_{2} v\left(e_{22}\right)$ \\
$\beta_{1}$ & $\gamma_{1}$ & 2 & $u\left(\hat{c}_{112}\right)\left(1+\beta_{1}\right)=u\left(c_{11}\right)+\gamma_{1} v\left(e_{11}\right)+\beta_{1} u\left(d_{11}\right)$ \\
$\beta_{1}$ & $\gamma_{2}$ & 2 & $u\left(\hat{c}_{122}\right)\left(1+\beta_{1}\right)=u\left(c_{12}\right)+\gamma_{2} v\left(e_{12}\right)+\beta_{1} u\left(d_{12}\right)$ \\
$\beta_{2}$ & $\gamma_{1}$ & 2 & $u\left(\hat{c}_{212}\right)\left(1+\beta_{2}\right)=u\left(c_{21}\right)+\gamma_{1} v\left(e_{21}\right)+\beta_{2} u\left(d_{21}\right)$ \\
$\beta_{2}$ & $\gamma_{2}$ & 2 & $u\left(\hat{c}_{222}\right)\left(1+\beta_{2}\right)=u\left(c_{22}\right)+\gamma_{2} v\left(e_{22}\right)+\beta_{2} u\left(d_{22}\right)$
\end{tabular}

The problem of the social planner can thus be written as follows:

$$
\max _{c_{i j}, d_{i j}, e_{i j}} \min \left(\hat{c}_{111}, \hat{c}_{121}, \hat{c}_{211}, \hat{c}_{221}, \hat{c}_{112}, \hat{c}_{122}, \hat{c}_{212}, \hat{c}_{222}\right) \quad \text { s.t. BC }
$$

where the CCPERLE $\hat{c}_{i j \ell}$ are defined in the above table.

Proposition 5 Under perfect information about ex-ante types $\left(\beta_{i}, \gamma_{j}\right)$, and $(4+2 \tilde{\pi}+2 \bar{\pi}) \bar{c} \leq W$, the Maximin CCPERLE optimum involves, under $e^{*}=0$ :

$$
\begin{aligned}
\bar{c} & <c_{12}^{*}<c_{11}^{*}, c_{22}^{*}<c_{21}^{*} \\
d_{11}^{*} & =d_{12}^{*}=d_{21}^{*}=d_{22}^{*}=\bar{c} \\
e_{11}^{*} & =e_{21}^{*}=0<e_{12}^{*}=e_{22}^{*}=\bar{e}
\end{aligned}
$$

Proof. See the Appendix.

There is a unique difference with respect to the baseline case where $e^{*}=\bar{e}$ : here effort-averse agents receive, at the young age, a higher consumption than effort-loving agents, instead of a lower consumption. The same difference holds with respect to the second-best Maximin CCPERLE, at which effort-loving agents have, under $e^{*}=\bar{e}$, a higher first-period consumption than effort-averse agents.

Turning now to the Sum of Transformed CCPERLE, the social planner's problem can still be rewritten, when we consider that $\ell^{*}=1$ and $e^{*}=\bar{e}$, as:

$$
\max _{c_{i j}, d_{i j}, e_{i j}} \sum_{i, j} n_{i j 1} h_{i j 1}\left(c_{i j}, e_{i j}\right)+\sum_{i, j} n_{i j 2} h_{i j 2}\left(c_{i j}, d_{i j}, e_{i j}\right) \quad \text { s.t. BC }
$$


where $h_{i j 1}\left(c_{i j}, e_{i j}\right) \equiv g \circ f_{i j 1}\left(c_{i j}, e_{i j}\right)$ and $h_{i j 2}\left(c_{i j}, d_{i j}, e_{i j}\right) \equiv g \circ f_{i j 2}\left(c_{i j}, d_{i j}, e_{i j}\right)$, with $f_{i j 1}\left(c_{i j}, e_{i j}\right) \equiv f\left(U_{i j}^{1}-\gamma_{j} v(\bar{e})\right)$ and $f_{i j 2}\left(c_{i j}, d_{i j}, e_{i j}\right) \equiv f\left(U_{i j}^{2}-\gamma_{j} v(\bar{e})\right)$.

Proposition 6 Under perfect information about ex-ante types $\left(\beta_{i}, \gamma_{j}\right)$, and $(4+4 \bar{\pi}) \bar{c} \leq W$, the Sum of Transformed CCPERLE optimum involves, un$\operatorname{der} \ell^{*}=1$ :

$$
\begin{aligned}
& c_{i 1}^{* *}<c_{i 2}^{* *} \forall i \\
& \left\{\begin{array}{c}
c_{1 j}^{* *}<c_{2 j}^{* *} \text { when low } \pi(\cdot) \\
c_{1 j}^{* *}>c_{2 j}^{* *} \text { when high } \pi(\cdot)
\end{array}\right. \\
& c_{i j}^{* *}>d_{i j}^{* *} \forall i, j \\
& d_{22}^{* *}>d_{12}^{* *}, d_{21}^{* *}>d_{11}^{* *}>\bar{c} \\
& e_{12}^{* *}=e_{22}^{* *}=\bar{e}>e_{21}^{* *}>e_{11}^{* *}>0
\end{aligned}
$$

Proof. See the Appendix.

Thus the Sum of Transformed CCPERLE social optimum looks, from a qualitative perspective, quite robust to the selected reference longevity.

Turning now to the alternative case where $\ell^{*}=2$ and $e^{*}=0$, the social planner problem becomes:

$$
\max _{c_{i j}, d_{i j}, e_{i j}} \sum_{i, j} n_{i j 1} h_{i j 1}\left(c_{i j}, e_{i j}\right)+\sum_{i, j} n_{i j 2} h_{i j 2}\left(c_{i j}, d_{i j}, e_{i j}\right) \quad \text { s.t. BC }
$$

where $h_{i j 1}\left(c_{i j}, e_{i j}\right) \equiv g \circ f_{i j 1}\left(c_{i j}, e_{i j}\right)$ and $h_{i j 2}\left(c_{i j}, d_{i j}, e_{i j}\right) \equiv g \circ f_{i j 2}\left(c_{i j}, d_{i j}, e_{i j}\right)$, with $f_{i j 1}\left(c_{i j}, e_{i j}\right) \equiv f\left(\frac{U_{i j}^{1}}{1+\beta_{i}}\right)$ and $f_{i j 2}\left(c_{i j}, d_{i j}, e_{i j}\right) \equiv f\left(\frac{U_{i j}^{2}}{1+\beta_{i}}\right)$.

Proposition 7 Under perfect information about ex-ante types $\left(\beta_{i}, \gamma_{j}\right)$, and $(4+4 \bar{\pi}) \bar{c} \leq W$, the Sum of Transformed CCPERLE optimum involves, un$\operatorname{der} e^{*}=0$ :

$$
\begin{aligned}
& c_{i 1}^{* *}>c_{i 2}^{* *} \forall i \\
& \left\{\begin{array}{c}
c_{1 j}^{* *}<c_{2 j}^{* *} \text { when low } \pi(\cdot) \\
c_{1 j}^{* *}>c_{2 j}^{* *} \text { when high } \pi(\cdot)
\end{array}\right. \\
& c_{i j}^{* *}>d_{i j}^{* *} \forall i, j \\
& d_{22}^{* *}>d_{12}^{* *}, d_{21}^{* *}>d_{11}^{* *}>\bar{c} \\
& e_{12}^{* *}=e_{22}^{* *}=\bar{e}>e_{21}^{* *}>e_{11}^{* *}>0
\end{aligned}
$$

Proof. See the Appendix.

The Sum of Transformed CCPERLE optimum involves here higher firstperiod consumptions for the effort-averse agents in comparison to the effortloving agents, contrary to what prevails when the reference effort is the maximum effort. The reason is that the change in the reference effort makes the marginal change in CCPERLE of short-lived effort-averse agents larger, ceteris paribus, than the one of short-lived effort-loving agents. 
In sum, although some changes may arise in terms of first-period consumption differentials across different ex ante types, both the Maximin CCPERLE and the Sum of Transformed CCPERLE are quite robust to changes in the reference levels of longevity and prevention effort.

\section{Conclusions}

Common sense supports prevention policies aimed at improving survival chances. But it is also widely acknowledged that a premature death is a serious disadvantage, which raises strong compensation concerns. Thus postponing death and making death benign constitute two reasonable attitudes in front of death.

Although both intuitive, those attitudes are logically incompatible. Indeed, we showed, by means of a simple model of risky lifetime with heterogeneous attitudes towards the future and towards prevention, that no social ordering on allocations can satisfy both a concern for prevention and a concern for compensation. The reason is that, if it is socially desirable to increase the number of survivors through prevention, it must also be, under costly prevention, desirable to deteriorate the living standards of the short-lived, which contradicts compensation. Hence a dilemma exists between prevention and compensation.

We proposed here two alternative approaches to that dilemma: either giving priority to compensation (i.e. Maximin CCPERLE), or giving up that priority, and favouring prevention (Sum of Transformed CCPERLE). We showed that the former social objective leads us to restrict prevention only to individuals who like it - whatever its effects on survival chances are - while effort-averse individuals should be left free to do no prevention. On the contrary, the latter objective, while still leading to a differentiated prevention on the basis of individual preferences, generally supports prevention for all.

Each of these two approaches to the compensation / prevention dilemma suffers from its own weaknesses. On the one hand, priority to compensation can only be achieved at the cost of worse survival chances. On the other hand, giving up priority to compensation will favour the survival of a larger number of individuals, but at the cost of even worse living standards for the unlucky short-lived. In any case, the dilemma remains, and, in that context, the optimal policy should be regarded not as the "best" policy, but, rather, as the "least bad" way to deal with that inevitable dilemma.

The goal of this paper is not to argue against prevention policies. From a historical perspective, prevention has played a major role in the secular improvement of survival conditions (see Easterlin 1999), and it is still, nowadays, a major instrument for the improvement of aggregate longevity outcomes. What we want to highlight here is rather that massive prevention, by trying to postpone death for all, can, for some persons, make premature death even more damageable than it would have been without prevention. It follows from this that a dilemma between prevention and compensation exists, and cannot be avoided. How a society deals with this dilemma is a matter of social choice. 


\section{References}

Atkinson, A.B. and J. Stiglitz, 1980. Lectures on Public Economics, McGraw-Hill International Editions, New-York.

Auster, R., Levenson, I. and D. Sarachek, 1969. 'The production of health: an exploratory study', Journal of Human Resources, 4: 411-436.

Balia, S. and A. Jones, 2008. 'Mortality, lifestyle and socio-economic status', Journal of Health Economics, 27(1): 1-26.

Becker, G., Philipson, T., and R. Soares, 2005. 'The quantity and the quality of life and the evolution of world inequality', American Economic Review, 95(1): 277-291.

Christensen, K., Johnson, T., and J. Vaupel, 2006. 'The quest for genetic determinants of human longevity: challenges and insights', Nature Reviews - Genetics, 7: 436-448.

Contoyannis, P. \& A. Jones, 2004. 'Socio-economic status, health and lifestyle', Journal of Health Economics, 23(5): 965-995.

Easterlin, R., 1999. 'How beneficent is the market? A look at the modern history of mortality', European Review of Economic History, 3 (3): 257-294.

Fleurbaey, M., 2008. Fairness, Responsibility and Welfare. New-York: Oxford University Press.

Fleurbaey, M., Leroux, M.L. \& Ponthiere, G., 2011. 'Compensating the dead', Princeton Economic Theory Center Working Paper 28-2011.

Fleurbaey, M. \& Maniquet, F., 2011, A Theory of Fairness and Social Welfare, Cambridge: Cambridge University Press.

Fong, G.T., Hammond, D., Laux, F., Zanna, M., Cummings, M., Borland, R. \& Ross, H., 2004. 'The near-universal experience of regret among smokers in four countries: findings from the International Tobacco Control Policy Evaluation Survey', Nicotine and Tobacco Research, 6(S3): S341-S351.

Hammond P.J. 1979, 'Equity in two-person situations', Econometrica, 47: 1127-1136.

Hansson, B., 1973. 'The independence condition in the theory of social choice', Theory and Decision, 25-49.

Jarvis, M., McIntyre, D., \& Bates, C. 2002. 'Effectiveness of smoking cessation initiatives', British Medical Journal, 324: 608.

Kaplan, G.A, T.E. Seeman, R.D. Cohen, L.P. Knudsen \& J. Guralnik, 1987. 'Mortality among the elderly in the Alameda county study: behavioral and demographic risk factors'. American Journal of Public Health, 77(3): 307-312.

Mullahy, J. \& P. Portney, 1990. 'Air pollution, cigarette smoking and the production of respiratory health', Journal of Health Economics, 9, 193-205.

Mullahy, J. \& J. Sindelar, 1996. 'Employment, unemployment, and problem drinking', Journal of Health Economics, 15, 409-434.

Pazner, E., 1979. 'Equity, nonfeasible alternatives and social choices: a reconsideration of the concept of social welfare', in J.J. Laffont (ed.): Aggregation and Revelation of Preferences, North Holland, Amsterdam.

Pazner, E. \& Schmeidler, D., 1978. 'Egalitarian equivalent allocations: a new concept of economic equity', Quarterly Journal of Economics, 92: 671-687.

Slovic, P., 2001. 'Cigarette smokers: rational actors or rational fools?', in P. Slovic (ed.) Smoking: Risk, perception, and policy, 97-126, Thousand Oaks, CA: Sage. 


\section{Appendix}

\subsection{Proof of Theorem 3}

Weak Pareto and Continuity imply that Strong Pareto is also satisfied.

By Pareto Indifference, it is enough to have a ranking of all allocations in which for all $i$, longevity is at the reference and consumption is constant. This ranking is then extended to all allocations in a unique way.

By Strong Pareto, Continuity, and Separability, and the Debreu-Gorman theorem, for every $R_{N}$ there is an increasing $\varphi_{i R_{N}}$, for every $i$, such that $x_{N} \succsim_{R_{N}} x_{N}^{\prime}$ iff $\sum_{i \in N} \varphi_{i R_{N}}\left(\hat{c}_{N}\right) \geq \sum_{i \in N} \varphi_{i R_{N}}\left(\hat{c}_{N}^{\prime}\right)$. By PDCCREL, $\varphi_{i R_{N}}$ must be concave and one can take the same $\varphi_{R_{N}}$ for every $i$.

Hansson Independence and Separability entail a stronger form of Separability:

Axiom 8 (Strong Separability) For all $R_{N}, R_{N}^{\prime} \in \Re^{|N|}$, all $x_{N}, x_{N}^{\prime} \in X^{|N|}$, all $M \subseteq N$,

$$
\left(x_{M}, x_{N \backslash M}\right) \succsim_{\left(R_{M}, R_{N \backslash M}\right)}\left(x_{M}^{\prime}, x_{N \backslash M}\right) \Leftrightarrow\left(x_{M}, x_{N \backslash M}^{\prime}\right) \succsim_{\left(R_{M}, R_{N \backslash M}^{\prime}\right)}\left(x_{M}^{\prime}, x_{N \backslash M}^{\prime}\right) .
$$

This implication is shown by considering $R_{N \backslash M}^{\prime \prime}, R_{N \backslash M}^{\prime \prime \prime}$ and $x_{N \backslash M}^{\prime \prime}$ such that indifference curves for $\left(x_{N \backslash M}, R_{N \backslash M}\right)$ coincide with those of $\left(x_{N \backslash M}, R_{N \backslash M}^{\prime \prime}\right)$, those of $\left(x_{N \backslash M}^{\prime}, R_{N \backslash M}^{\prime}\right)$ coincide with those of $\left(x_{N \backslash M}^{\prime}, R_{N \backslash M}^{\prime \prime \prime}\right)$, and those of $\left(x_{N \backslash M}^{\prime \prime}, R_{N \backslash M}^{\prime \prime}\right)$ coincide with those of $\left(x_{N \backslash M}^{\prime \prime}, R_{N \backslash M}^{\prime \prime \prime}\right)$. (This is done by taking a high $x_{N \backslash M}^{\prime \prime}$. Combining Hansson Independence and Separability, one gets

$$
\begin{aligned}
\left(x_{M}, x_{N \backslash M}\right) & \succsim \quad\left(R_{M}, R_{N \backslash M}\right)\left(x_{M}^{\prime}, x_{N \backslash M}\right) \Leftrightarrow\left(x_{M}, x_{N \backslash M}\right) \succsim_{\left(R_{M}, R_{N \backslash M}^{\prime \prime}\right)}\left(x_{M}^{\prime}, x_{N \backslash M}\right) \\
& \Leftrightarrow \quad\left(x_{M}, x_{N \backslash M}^{\prime \prime}\right) \succsim_{\left(R_{M}, R_{N \backslash M}^{\prime \prime}\right)}\left(x_{M}^{\prime}, x_{N \backslash M}^{\prime \prime}\right) \\
& \Leftrightarrow \quad\left(x_{M}, x_{N \backslash M}^{\prime \prime}\right) \succsim_{\left(R_{M}, R_{N \backslash M}^{\prime}\right)}\left(x_{M}^{\prime}, x_{N \backslash M}^{\prime \prime}\right) \\
& \Leftrightarrow \quad\left(x_{M}, x_{N \backslash M}^{\prime}\right) \succsim_{\left(R_{M}, R_{N \backslash M}^{\prime}\right.}\left(x_{M}^{\prime}, x_{N \backslash M}^{\prime}\right) .
\end{aligned}
$$

This Strong Separability axiom implies that one can take the same $\varphi$ for all $R_{N}$ (because it implies that $\varphi_{R_{N}}$ does not depend on $R_{N \backslash M}$ for all $M$ ). It is then straightforward to check that $\sum_{i \in N} \varphi\left(\hat{c}_{N}\right)$ satisfies Survivors Numbers Count.

\subsection{Proof of Proposition 1}

To find the bundles maximizing the minimum CCPERLE, we first need to identify the worst-off agents. We can first note that, if $u\left(d_{i j}\right)>0$, the short-lived individuals are worse-off than the long-lived individuals with the same ex ante 
characteristics. Alternatively, if $u\left(d_{i j}\right)<0$, the short-lived are better-off than the long-lived individuals, ceteris paribus. Hence the perfect equality of lifetime welfare across individuals with the same $e x$ ante characteristics involves: ${ }^{23}$

$$
d_{11}^{*}=d_{21}^{*}=d_{12}^{*}=d_{22}^{*}=\bar{c}
$$

Moreover, it appears also clearly from the table that the CCPERLE of all shortlived agents can be raised by imposing the minimum level of effort to those who do not like effort, and the maximum level of effort to those who like it:

$$
e_{11}^{*}=e_{21}^{*}=0<e_{12}^{*}=e_{22}^{*}=\bar{e}
$$

Hence, three cases can arise, depending on the level of endowment $W$.

- If $(4+2 \tilde{\pi}+2 \bar{\pi}) \bar{c} \leq W$, the optimum involves:

$$
\begin{aligned}
& d_{11}^{*}=d_{12}^{*}=d_{21}^{*}=d_{22}^{*}=\bar{c} \\
& c_{11}^{*}, c_{12}^{*}, c_{21}^{*}, c_{22}^{*}>\bar{c}
\end{aligned}
$$

- If $(2 \tilde{\pi}+2 \bar{\pi}) \bar{c} \leq W \leq(4+2 \tilde{\pi}+2 \bar{\pi}) \bar{c}$, the optimum involves:

$$
\begin{aligned}
& d_{11}^{*}=d_{12}^{*}=d_{21}^{*}=d_{22}^{*}=\bar{c} \\
& c_{11}^{*}, c_{12}^{*}, c_{21}^{*}, c_{22}^{*} \leq \bar{c}
\end{aligned}
$$

- If $W \leq(2 \tilde{\pi}+2 \bar{\pi}) \bar{c}$, the optimum involves:

$$
\begin{aligned}
& d_{11}^{*}<c_{11}^{*}<\bar{c} \text { and } d_{12}^{*}<c_{12}^{*}<\bar{c} \\
& d_{21}^{*}<c_{21}^{*}<\bar{c} \text { and } d_{22}^{*}<c_{22}^{*}<\bar{c}
\end{aligned}
$$

In rich economies, the worst-offs are the short-lived, and compensation requires welfare-neutral consumption at the old age, while the young enjoy many more resources. In a poor economy, it is rather the opposite: the long-lived are the worst-offs, and compensation requires to give to the long-lived the welfareneutral consumption, and less to the young.

Let us now focus on consumption at the young age. If one compares then short-lived agents of type- $i 1$ with short-lived agents of type- $i 2$, it is clear that, given $e_{i 1}=0<e_{i 2}=\bar{e}$, short-lived type- $i 1$ have always a higher CCPERLE than short-lived type- $i 2$ when first-period consumptions are equalized. ${ }^{24}$ Furthermore, if one compares two agents with an equal $\gamma_{j}$ but different $\beta_{i}$, it

\footnotetext{
${ }^{23}$ That strategy is actually quite similar to what used to prevail in the absence of healthaffecting effort (see Fleurbaey et al 2011).

${ }^{24}$ To see this, focus on the first two types. After simplification for effort levels, we have:

$$
\begin{aligned}
u\left(\hat{c}_{111}\right)\left(1+\beta_{1}\right)+\gamma_{1}\left[v(\bar{e})-v\left(e_{11}\right)\right] & =u\left(c_{11}\right) \\
u\left(\hat{c}_{121}\right)\left(1+\beta_{1}\right) & =u\left(c_{12}\right)
\end{aligned}
$$
}

Given that $\gamma_{1}\left[v(\bar{e})-v\left(e_{11}\right)\right]<0$, it follows that $\hat{c}_{111}>\hat{c}_{121}$ for an equal consumption level. Focusing on the next two types, we have:

$$
\begin{aligned}
u\left(\hat{c}_{211}\right)\left(1+\beta_{2}\right)+\gamma_{1}\left[v(\bar{e})-v\left(e_{21}\right)\right] & =u\left(c_{21}\right) \\
u\left(\hat{c}_{221}\right)\left(1+\beta_{2}\right) & =u\left(c_{22}\right)
\end{aligned}
$$

yielding here again $\hat{c}_{211}>\hat{c}_{221}$ under equal consumptions. 
appears that type- $2 j$ agents have a lower CCPERLE than type- $1 j$ agents for the same level of effort. Hence the worst-off agents who should benefit from priority are clearly type-22 agents, who are patient and like effort. Actually, under equal first-period consumptions, we would have:

$$
\hat{c}_{221}<\hat{c}_{121}<\hat{c}_{111} \quad \text { and } \quad \hat{c}_{221}<\hat{c}_{211}<\hat{c}_{111} \quad \text { and } \quad \hat{c}_{121} \gtrless \hat{c}_{211}
$$

Therefore, the equalization of the CCPERLE among the short-lived requires:

$$
c_{11}^{*}<c_{12}^{*}<c_{22}^{*} \quad \text { and } \quad c_{11}^{*}<c_{21}^{*}<c_{22}^{*} \quad \text { and } \quad c_{12}^{*} \gtrless c_{21}^{*}
$$

Hence, first-period consumption is the largest for patient agents who like efforts (i.e. $c_{22}^{*}$ ), and the smallest for impatient agents who do not like efforts (i.e. $c_{11}^{*}$ ). For an equal $\gamma_{j}$, the patient receive a higher first-period consumption than the impatient, whereas, for equal $\beta_{i}$, the agents who like effort receive a higher first-period consumption than those who dislike effort. However, once we start comparing agents differing in both patience and attitude towards effort, no obvious ranking of CCPERLE can be found. ${ }^{25}$

Let us now compute the CCPERLE ranking at the optimum. Here again, several cases can arise.

- If $(4+2 \tilde{\pi}+2 \bar{\pi}) \bar{c} \leq W$, the optimum involves:

$$
\begin{aligned}
& d_{11}^{*}=d_{12}^{*}=d_{21}^{*}=d_{22}^{*}=\bar{c} \\
& \bar{c}<c_{11}^{*}<c_{12}^{*}<c_{22}^{*} \quad \text { and } \quad c_{11}^{*}<c_{21}^{*}<c_{22}^{*} \quad \text { and } \quad c_{12}^{*} \gtrless c_{21}^{*}
\end{aligned}
$$

From which we may obtain a perfect equalization of CCPERLE levels:

$$
\hat{c}_{111}=\hat{c}_{121}=\hat{c}_{211}=\hat{c}_{221}=\hat{c}_{112}=\hat{c}_{122}=\hat{c}_{212}=\hat{c}_{222}
$$

or keeping some inequalities may remain across ex ante types (when the welfare gap due to different efforts cannot be compensated by unequal consumptions at the young age):

$$
\begin{aligned}
& \hat{c}_{121}=\hat{c}_{122} \leq \hat{c}_{111}=\hat{c}_{112} \quad \text { and } \quad \hat{c}_{221}=\hat{c}_{222} \leq \hat{c}_{211}=\hat{c}_{212} \\
& \hat{c}_{211}=\hat{c}_{212} \leq \hat{c}_{111}=\hat{c}_{112} \quad \text { and } \quad \hat{c}_{221}=\hat{c}_{222} \leq \hat{c}_{121}=\hat{c}_{122}
\end{aligned}
$$

- If $(2 \tilde{\pi}+2 \bar{\pi}) \bar{c} \leq W \leq(4+2 \tilde{\pi}+2 \bar{\pi}) \bar{c}$, the optimum involves also a perfect equalization of CCPERLE between individuals with the same ex ante characteristics, but some inequalities may remain across ex ante types:

$$
\begin{aligned}
& \hat{c}_{121}=\hat{c}_{122} \leq \hat{c}_{111}=\hat{c}_{112} \quad \text { and } \quad \hat{c}_{221}=\hat{c}_{222} \leq \hat{c}_{211}=\hat{c}_{212} \\
& \hat{c}_{211}=\hat{c}_{212} \leq \hat{c}_{111}=\hat{c}_{112} \quad \text { and } \quad \hat{c}_{221}=\hat{c}_{222} \leq \hat{c}_{121}=\hat{c}_{122}
\end{aligned}
$$

\footnotetext{
${ }^{25}$ The reason is that patient agents who dislike effort may make more or less effort than impatient agents who like effort, so that the optimal consumptions $c_{12}^{*}$ and $c_{21}^{*}$ cannot be ranked, but are necessarily between $c_{11}^{*}$ and $c_{22}^{*}$.
} 
- If $W \leq(2 \tilde{\pi}+2 \bar{\pi}) \bar{c}$, the optimum involves inequalities between short-lived and long-lived within a given ex ante type, and may also involve other inequalities across ex ante types:

$$
\begin{aligned}
& \hat{c}_{122}<\hat{c}_{121} \leq \hat{c}_{112}<\hat{c}_{111} \quad \text { and } \quad \hat{c}_{222}<\hat{c}_{221} \leq \hat{c}_{212}<\hat{c}_{211} \\
& \hat{c}_{212}<\hat{c}_{211} \leq \hat{c}_{112}<\hat{c}_{111} \quad \text { and } \quad \hat{c}_{222}<\hat{c}_{221} \leq \hat{c}_{122}<\hat{c}_{121}
\end{aligned}
$$

Thus, the introduction of health-affecting efforts may prevent the perfect equalization of lifetime welfare across all individuals, even when a full compensation for unequal longevities can be provided. The reason is that welfare inequalities at the young age depend strongly on the shape of the disutility of effort $v(e)$ in comparison with the shape of the utility of consumption $u(c) .{ }^{26}$

\subsection{Properties of the CCPERLE}

Let us check the relative relevance of Cases A and B by taking a simple analytical example, with:

$$
U_{i j}^{1}=\left(c_{i j}\right)^{\epsilon}+\gamma_{j} \frac{\left(e_{i j}\right)^{2}}{2}, U_{i j}^{2}=\left(c_{i j}\right)^{\epsilon}+\gamma_{j} \frac{\left(e_{i j}\right)^{2}}{2}+\beta_{i}\left(d_{i j}\right)^{\epsilon},
$$

where $0<\epsilon<1$. In that case, the (des)utility of effort is quadratic.

Hence the CCPERLE is here:

$$
\hat{c}_{i j \ell}=\left(\frac{U_{i j}^{\ell}-\gamma_{j} \frac{(\bar{e})^{2}}{2}}{1+\beta_{i}}\right)^{\frac{1}{\epsilon}}
$$

Thus we have:

$\hat{c}_{i j 1}=\left(\frac{\left(c_{i j}\right)^{\epsilon}+\gamma_{j} \frac{\left(e_{i j}\right)^{2}}{2}-\gamma_{j} \frac{(\bar{e})^{2}}{2}}{1+\beta_{i}}\right)^{\frac{1}{\epsilon}}, \hat{c}_{i j 2}=\left(\frac{\left(c_{i j}\right)^{\epsilon}+\beta_{i}\left(d_{i j}\right)^{\epsilon}+\gamma_{j} \frac{\left(e_{i j}\right)^{2}}{2}-\gamma_{j} \frac{(\bar{e})^{2}}{2}}{1+\beta_{i}}\right)^{\frac{1}{\epsilon}}$

Hence the derivatives of the untransformed CCPERLE are here:

$$
\begin{aligned}
& \frac{\partial \hat{c}_{i 11}}{\partial c_{i 1}}>0, \frac{\partial \hat{c}_{i 21}}{\partial c_{i 2}}>0 ; \frac{\partial \hat{c}_{i 11}^{2}}{\partial c_{i 1}^{2}}<0, \frac{\partial \hat{c}_{i 21}^{2}}{\partial c_{i 2}^{2}}=0 \\
& \frac{\partial \hat{c}_{i 12}}{\partial c_{i 1}}>0, \frac{\partial \hat{c}_{i 22}}{\partial c_{i 2}}>0 ; \frac{\partial \hat{c}_{i 12}^{2}}{\partial c_{i 1}^{2}}<0, \frac{\partial \hat{c}_{i 22}^{2}}{\partial c_{i 2}^{2}}<0 \\
& \frac{\partial \hat{c}_{i 12}}{\partial d_{i 1}}>0, \frac{\partial \hat{c}_{i 22}}{\partial d_{i 2}}>0 ; \frac{\partial \hat{c}_{i 12}^{2}}{\partial d_{i 1}^{2}}<0, \frac{\partial \hat{c}_{i 22}^{2}}{\partial d_{i 2}^{2}}<0 \\
& \frac{\partial \hat{c}_{i 11}}{\partial e_{i 1}}<0, \frac{\partial \hat{c}_{i 21}}{\partial e_{i 2}}>0 ; \frac{\partial \hat{c}_{i 11}^{2}}{\partial e_{i 1}^{2}} \lessgtr 0, \frac{\partial \hat{c}_{i 21}^{2}}{\partial e_{i 2}^{2}}>0 \\
& \frac{\partial \hat{c}_{i 12}}{\partial e_{i 1}}<0, \frac{\partial \hat{c}_{i 22}}{\partial e_{i 2}}>0 ; \frac{\partial \hat{c}_{i 12}^{2}}{\partial e_{i 1}^{2}} \lessgtr 0, \frac{\partial \hat{c}_{i 22}^{2}}{\partial e_{i 2}^{2}}>0
\end{aligned}
$$

\footnotetext{
${ }^{26}$ If $u(c)$ is too concave, increasing the consumption of those who hate effort may not allow a full compensation of disutility from efforts, and so some welfare inequality may remain between ex ante types.
} 


\subsection{Proof of Proposition 2}

Defining $\lambda$ as the Lagrange multiplier associated with the resource constraint of the economy, the first-order conditions of the social planner's problem yield:

$$
\begin{aligned}
\left(1-\pi\left(e_{11}^{* *}\right)\right) \frac{\partial h_{111}(\cdot)}{\partial c_{11}^{* *}}+\pi\left(e_{11}^{* *}\right) \frac{\partial h_{112}(\cdot)}{\partial c_{11}^{* *}} & =\lambda=\frac{\partial h_{112}(\cdot)}{\partial d_{11}^{* *}} \\
\left(1-\pi\left(e_{12}^{* *}\right)\right) \frac{\partial h_{121}(\cdot)}{\partial c_{12}^{* *}}+\pi\left(e_{12}^{* *}\right) \frac{\partial h_{122}(\cdot)}{\partial c_{12}^{* *}} & =\lambda=\frac{\partial h_{122}(\cdot)}{\partial d_{12}^{* *}} \\
\left(1-\pi\left(e_{21}^{* *}\right)\right) \frac{\partial h_{211}(\cdot)}{\partial c_{21}^{* *}}+\pi\left(e_{21}^{* *}\right) \frac{\partial h_{212}(\cdot)}{\partial c_{21}^{* *}} & =\lambda=\frac{\partial h_{212}(\cdot)}{\partial d_{21}^{* *}} \\
\left(1-\pi\left(e_{22}^{* *}\right)\right) \frac{\partial h_{221}(\cdot)}{\partial c_{22}^{* *}}+\pi\left(e_{22}^{* *}\right) \frac{\partial h_{222}(\cdot)}{\partial c_{22}^{* *}} & =\lambda=\frac{\partial h_{222}(\cdot)}{\partial d_{22}^{* *}} \\
\pi^{\prime}\left(e_{11}^{* *}\right)\left[h_{112}(\cdot)-h_{111}(\cdot)-\frac{\partial h_{112}(\cdot)}{\partial d_{11}^{* *}} d_{11}^{* *}\right] & =-\left[\left(1-\pi\left(e_{11}^{* *}\right)\right) \frac{\partial h_{111}(\cdot)}{\partial e_{11}^{* *}}+\pi\left(e_{11}^{* *}\right) \frac{\partial h_{112}(\cdot)}{\partial e_{11}^{* *}}\right]>0 \\
\pi^{\prime}\left(e_{12}^{* *}\right)\left[h_{122}(\cdot)-h_{121}(\cdot)-\frac{\partial h_{122}(\cdot)}{\partial d_{12}^{* *}} d_{12}^{* *}\right] & =-\left[\left(1-\pi\left(e_{12}^{* *}\right)\right) \frac{\partial h_{121}(\cdot)}{\partial e_{12}^{* *}}+\pi\left(e_{12}^{* *}\right) \frac{\partial h_{122}(\cdot)}{\partial e_{12}^{* *}}\right]<0 \\
\pi^{\prime}\left(e_{21}^{* *}\right)\left[h_{212}(\cdot)-h_{211}(\cdot)-\frac{\partial h_{212}(\cdot)}{\partial d_{21}^{* *}} d_{21}^{* *}\right] & =-\left[\left(1-\pi\left(e_{21}^{* *}\right)\right) \frac{\partial h_{211}(\cdot)}{\partial e_{21}^{* *}}+\pi\left(e_{21}^{* *}\right) \frac{\partial h_{212}(\cdot)}{\partial e_{21}^{* *}}\right]>0 \\
\pi^{\prime}\left(e_{22}^{* *}\right)\left[h_{222}(\cdot)-h_{221}(\cdot)-\frac{\partial h_{222}(\cdot)}{\partial d_{22}^{* *}} d_{22}^{* *}\right] & =-\left[\left(1-\pi\left(e_{22}^{* *}\right)\right) \frac{\partial h_{221}(\cdot)}{\partial e_{22}^{* *}}+\pi\left(e_{22}^{* *}\right) \frac{\partial h_{222}(\cdot)}{\partial e_{22}^{* *}}\right]<0
\end{aligned}
$$

Assuming that resources are sufficient to make survival desirable (i.e. $(4+4 \bar{\pi}) \bar{c} \leq$ $W$ ), the LHS of the FOCs for optimal prevention is necessarily positive, implying that the second and fourth FOC are not be satisfied: in the case of effort-loving agents, the socially optimal effort level is a corner solution, equal to the maximum effort: $e_{12}^{* *}=e_{22}^{* *}=\bar{e}$. Moreover, given that the net gain, in terms of the transformed CCPERLE, associated with survival is, in general, higher for patient agents than impatient agents (so that the factor in brackets in the LHS is larger for patient agents), whereas the welfare cost of effort is the same, we expect, assuming an interior social optimum, it is optimal that $\beta_{2}$-type agents make more efforts, ceteris paribus, than $\beta_{1}$-type agents. Hence we obtain: $e_{12}^{* *}=e_{22}^{* *}=\bar{e}>e_{21}^{* *}>e_{11}^{* *}>0$.

Let us now combine, for a given type $i j$, the FOCs for optimal consumption at the young age and at the old age: $\frac{\partial h_{i j 1}(\cdot)}{\partial c_{i j}^{* * *}}-\pi\left(e_{i j}^{* *}\right) \frac{\partial h_{i j 1}(\cdot)}{\partial c_{i j}^{* * *}}=\frac{\partial h_{i j 2}(\cdot)}{\partial d_{i j}^{* * *}}-$ $\pi\left(e_{i j}^{* *}\right) \frac{\partial h_{i j 2}(\cdot)}{\partial c_{i j}^{* *}}$. Given that $h \equiv g \circ f\left(\frac{U_{i j}^{\ell}-\gamma_{j} v(\bar{e})}{1+\beta_{i}}\right)$ is concave, we have that $\frac{\partial h_{i j 1}(\cdot)}{\partial c_{i j}^{* *}}>\frac{\partial h_{i j 2}(\cdot)}{\partial c_{i j}^{* *}}$ ceteris paribus. Hence, the equality requires $\frac{\partial h_{i j 1}(\cdot)}{\partial c_{i j}^{* * *}}>\frac{\partial h_{i j 2}(\cdot)}{\partial d_{i j}^{* *}}$, that is, the effect, on the transformed CCPERLE of a short-lived agent, of a marginal rise in $c_{i j}$ must, at the optimum, exceed the effect, on the transformed equivalent for a long-lived agent, of a marginal rise in $d_{i j}$. Such an inequality rules out, given the concavity of $u(\cdot)$, cases where $d_{i j}$ is too low. Hence we have: 
$d_{i j}^{* *}>\bar{c}^{27}$

Note also that: $\frac{\partial h_{i j 1}(\cdot)}{\partial c_{i j}^{* *}}+\pi\left(e_{i j}^{* *}\right)\left[\frac{\partial h_{i j 2}(\cdot)}{\partial c_{i j}^{* *}}-\frac{\partial h_{i j 1}(\cdot)}{\partial c_{i j}^{* *}}\right]=\frac{\partial h_{i j 2}(\cdot)}{\partial d_{i j}^{* *}}$. Hence, given that $\frac{\partial h_{i j 2}(\cdot)}{\partial c_{i j}^{* *}}-\frac{\partial h_{i j 1}(\cdot)}{\partial c_{i j}^{* *}}<0$, it follows that: $\frac{\partial h_{i j 1}(\cdot)}{\partial c_{i j}^{* *}}>\frac{\partial h_{i j 2}(\cdot)}{\partial d_{i j}^{* *}}$, implying $c_{i j}^{* *}>d_{i j}^{* *}$, that is, declining consumption profiles along the lifecycle.

Regarding second-period consumption, we also have: $\frac{\partial h_{112}(\cdot)}{\partial d_{11}^{* *}}=\frac{\partial h_{122}(\cdot)}{\partial d_{12}^{* *}}=$ $\frac{\partial h_{212}(\cdot)}{\partial d_{21}^{* *}}=\frac{\partial h_{222}(\cdot)}{\partial d_{22}^{* *}}$. Here again, given the concavity of $h(\cdot)$, and given that patient agents have, ceteris paribus, a higher marginal gain from second-period consumption, we expect $d_{2 j}^{* *}>d_{1 j}^{* *}$. The same is true, at the optimum, for effortloving agents, so that we expect $d_{i 2}^{* *}>d_{i 1}^{* *}$. Hence we have: $d_{22}^{* *}>d_{12}^{* *}, d_{21}^{* *}>$ $d_{11}^{* *}>\bar{c}$.

Let us now compare first-period consumption levels. We have: $\frac{\partial h_{111}(\cdot)}{\partial c_{11}^{* *}}+$ $\pi\left(e_{11}^{* *}\right)\left[\frac{\partial h_{112}(\cdot)}{\partial c_{11}^{* *}}-\frac{\partial h_{111}(\cdot)}{\partial c_{11}^{* *}}\right]=\frac{\partial h_{211}(\cdot)}{\partial c_{21}^{* *}}+\pi\left(e_{21}^{* *}\right)\left[\frac{\partial h_{212}(\cdot)}{\partial c_{21}^{* *}}-\frac{\partial h_{211}(\cdot)}{\partial c_{21}^{* *}}\right]$. Given the concavity of $h(\cdot)$, the effect, on the transformed CCPERLE, of a rise in firstperiod consumption in case of premature death is larger for $\beta_{2}$-type agents than for $\beta_{1}$-type agents. Hence $\frac{\partial h_{111}(\cdot)}{\partial c_{11}^{* *}}<\frac{\partial h_{211}(\cdot)}{\partial c_{21}^{* *}}$. Note that the marginal effect, on the transformed CCPERLE, of a rise in $c_{i j}$ is always larger for short-lived than for long-lived. Thus, the second terms of the LHS and RHS are negative. Moreover, given $d_{21}^{* *}>d_{11}^{* *}$, the effect, on the transformed CCPERLE, of a rise in first-period consumption in case of long life is larger for $\beta_{1}$-type agents than for $\beta_{2}$-type agents: $\frac{\partial h_{112}(\cdot)}{\partial c_{11}^{* *}}>\frac{\partial h_{212}(\cdot)}{\partial c_{21}^{* *}}$. Thus the first term of the RHS exceeds the first term of the LHS ceteris paribus, and, in absolute value, the factor in brackets of the LHS is larger than the one of the RHS. Note that, as $e_{11}^{* *}<e_{21}^{* *}$, more weight is assigned to the (negative) second term of the RHS than to the (negative) second term on the LHS. Thus the result depends on how large survival chances are.

- If low survival chances, i.e. a low $\pi(\cdot)$ for all effort levels, we have: $c_{21}^{* *}>$ $c_{11}^{* *}$, on the grounds of the larger welfare loss due to a shorter life in case of patient agents, even though that loss is less likely than for impatient agents, who make less effort.

- If high survival chances, i.e. a high $\pi(\cdot)$ for all effort levels, then we have: $c_{21}^{* *}<c_{11}^{* *}$, on the grounds of the more likely welfare loss for impatient agents, as these make less effort, even though that loss is less sizeable than for patient agents.

Regarding other first-period consumption levels, we have:

$$
\frac{\partial h_{111}(\cdot)}{\partial c_{11}^{* *}}+\pi\left(e_{11}^{* *}\right)\left[\frac{\partial h_{112}(\cdot)}{\partial c_{11}^{* * *}}-\frac{\partial h_{111}(\cdot)}{\partial c_{11}^{* *}}\right]=\frac{\partial h_{121}(\cdot)}{\partial c_{12}^{* *}}+\pi\left(e_{12}^{* *}\right)\left[\frac{\partial h_{122}(\cdot)}{\partial c_{12}^{* *}}-\frac{\partial h_{121}(\cdot)}{\partial c_{12}^{* *}}\right]
$$

${ }^{27}$ Indeed, if $d_{i j}^{* *}$ was lower than $\bar{c}$, we would get:

$$
\frac{\partial h_{i j 1}(\cdot)}{\partial c_{i j}}<\left.\frac{\partial h_{i j 2}(\cdot)}{\partial d_{i j}}\right|_{d_{i j}=\bar{c}}
$$

hence contradicting the above FOC. 
In case of short life, the marginal gain from first period consumption is larger for agents who have, ceteris paribus, the lowest CCPERLE, i.e. agents who like effort. Hence $\frac{\partial h_{111}(\cdot)}{\partial c_{11}^{* *}}<\frac{\partial h_{121}(\cdot)}{\partial c_{12}^{* *}}$ ceteris paribus. In case of long-lived agents, we also have $\frac{\partial h_{112}(\cdot)}{\partial c_{11}^{* *}}<\frac{\partial h_{122}(\cdot)}{\partial c_{12}^{* *}}$. However, given that the marginal effect, on the transformed CCPERLE, of a rise in $c_{i j}$ is always larger for short-lived than for long-lived, the terms in brackets are negative. Note, however, that, as $\frac{\partial h_{111}(\cdot)}{\partial c_{11}^{* *}}<\frac{\partial h_{121}(\cdot)}{\partial c_{12}^{* *}}$ and $\frac{\partial h_{112}(\cdot)}{\partial c_{11}^{* *}}<\frac{\partial h_{122}(\cdot)}{\partial c_{12}^{* *}}$, the two factors in brackets are likely to be very close to each others. Hence, the first terms will drive the optimal allocation of resources at the young age. Given $\frac{\partial h_{111}(\cdot)}{\partial c_{11}^{* *}}<\frac{\partial h_{121}(\cdot)}{\partial c_{12}^{* *}}$, we obtain that: $c_{11}^{* *}<c_{12}^{* *}$, since short-lived agents who like effort have larger marginal welfare gains from more consumption, in comparison to effort-averse agents. Following a similar rationale, we obtain also: $c_{21}^{* *}<c_{22}^{* *}$.

\subsection{Proof of Proposition 3}

Equal old-age consumption is trivially incentive compatible, as it will not induce any mimicking behavior. Let us now show that unequal consumption for patient and impatient agents at the young age is not incentive compatible. Incentive compatibility requires:

$u\left(c_{1 j}\right)+\gamma_{j} v\left(e_{1 j}\right)+\pi\left(e_{1 j}\right) \beta_{1} u\left(d_{1 j}\right) \geq u\left(c_{2 j}\right)+\gamma_{j} v\left(e_{2 j}\right)+\pi\left(e_{2 j}\right) \beta_{1} u\left(d_{2 j}\right) \quad \forall j=1,2$

Hence, if, like at the first-best, $d_{i j}=\bar{c}$ for all, as well as $e_{1 i}=e_{2 i}$, and if $c_{1 j}<c_{2 j}$, we obtain that the first term of the LHS is smaller than the first term of the RHS, while the second and third terms of the LHS are exactly equal to the ones of the RHS. Hence, incentive compatibility is not satisfied. Impatient agents have thus an incentive to lie on their type. To avoid this, the unique solution is to equalize first-period consumption for patient and impatient agents: $c_{1 j}=c_{2 j}$.

Turning now to the second incentive compatibility constraint, i.e.

$u\left(c_{i 1}\right)+\gamma_{1} v\left(e_{i 1}\right)+\pi\left(e_{i 1}\right) \beta_{i} u\left(d_{i 1}\right) \geq u\left(c_{i 2}\right)+\gamma_{1} v\left(e_{i 2}\right)+\pi\left(e_{i 2}\right) \beta_{i} u\left(d_{i 2}\right) \quad \forall i=1,2$

we see that, if, like in the first-best, we propose $d_{i j}=\bar{c}$, as well as $c_{i 1}<c_{i 2}$ and $e_{i 1}=0<e_{i 2}=\bar{e}$, we have:

$$
u\left(c_{i 1}\right) \geq u\left(c_{i 2}\right)+\gamma_{1} v(\bar{e}) \quad \forall i=1,2
$$

The LHS is smaller than the first-term of the RHS, and the second term of the RHS is negative. Hence, given that $\gamma_{1} v(\bar{e})$ is far below 0 , we have, in general, that the first-best inequality $c_{i 1}^{* *}<c_{i 2}^{* *}$ is incentive compatible.

\subsection{Proof of Proposition 4}

Changing the reference longevity level from $\ell^{*}=2$ to $\ell^{*}=1$ does not change the optimal level of second-period consumptions. Indeed, in order to obtain an 
equalization of lifetime welfare between long-lived and short-lived of a given $e x$ ante type, it is necessary, when resources are sufficiently large (i.e. $(4+2 \tilde{\pi}+2 \bar{\pi}) \bar{c} \leq$ $W$ ), to fix $d_{i j}^{*}=\bar{c} \forall i, j$. Moreover, regarding optimal prevention, we still obtain that effort-lovers should do the maximum, i.e. $e_{i 2}^{*}=\bar{e}$, while effort-averse agents should do the minimum: $e_{i 1}^{*}=0$. The only difference concerns firstperiod consumption. When comparing the CCPERLE of short-lived patient and impatient agents, we see that it is now independent from time preferences. This implies equal first-period consumption for patient and impatient agents: $c_{1 j}^{*}=c_{2 j}^{*}$. On the contrary, effort-loving agents still receive a higher first-period consumption, since their CCPERLE is lower than the one of effort-averse agents, ceteris paribus. This implies: $c_{i 1}^{*}<c_{i 2}^{*}$.

\subsection{Proof of Proposition 5}

Changing the reference effort level from $e^{*}=\bar{e}$ to $e^{*}=0$ does not change the optimal level of second-period consumptions. ${ }^{28}$ Moreover, regarding optimal prevention, we still obtain that effort-lovers should do the maximum, i.e. $e_{i 2}^{*}=\bar{e}$, while effort-averse agents should do the minimum: $e_{i 1}^{*}=0$. The only difference concerns first-period consumption. When comparing the CCPERLE of shortlived effort-averse and effort-loving agents, we see that the CCPERLE of the former is always smaller than the CCPERLE of the latter. This implies higher first-period consumption for effort-averse than for effort-loving agents ceteris paribus: $c_{i 1}^{*}>c_{i 2}^{*}$. Note that the CCPERLE of short-lived patient agents is still lower than the one of short-lived impatient agents, which implies: $c_{1 j}^{*}<c_{2 j}^{*}$.

\subsection{Proof of Proposition 6}

The FOCs of the social planner's problem are similar to the ones under the different definition of the CCPERLE. The difference is that, in the present context, $h_{i j 1}\left(c_{i j}, e_{i j}\right) \equiv g \circ f_{i j 1}\left(c_{i j}, e_{i j}\right)$ and $h_{i j 2}\left(c_{i j}, d_{i j}, e_{i j}\right) \equiv g \circ f_{i j 2}\left(c_{i j}, d_{i j}, e_{i j}\right)$, where $f\left(U_{i j}^{1}-\gamma_{j} v(\bar{e})\right) \equiv f_{i j 1}\left(c_{i j}, e_{i j}\right)$ and $f\left(U_{i j}^{2}-\gamma_{j} v(\bar{e})\right) \equiv f_{i j 2}\left(c_{i j}, d_{i j}, e_{i j}\right)$.

From the last four FOCs, we obtain, as above: $e_{12}^{* *}=e_{22}^{* *}=\bar{e}>e_{21}^{* *}>e_{11}^{* *}>$ 0 .

Let us now combine, for a given type $i j$, the FOCs for optimal consumption at the young age and at the old age: $\frac{\partial h_{i j 1}(\cdot)}{\partial c_{i j}^{* *}}-\pi\left(e_{i j}^{* *}\right) \frac{\partial h_{i j 1}(\cdot)}{\partial c_{i j}^{* *}}=\frac{\partial h_{i j 2}(\cdot)}{\partial d_{i j}^{* *}}-$ $\pi\left(e_{i j}^{* *}\right) \frac{\partial h_{i j 2}(\cdot)}{\partial c_{i j}^{* *}}$. Given that $h \equiv g \circ f\left(U_{i j}^{\ell}-\gamma_{j} v(\bar{e})\right)$ is concave, we have that $\frac{\partial h_{i j 1}(\cdot)}{\partial c_{i j}^{* *}}>\frac{\partial h_{i j 2}(\cdot)}{\partial c_{i j}^{* *}}$ ceteris paribus. Hence, the equality requires $\frac{\partial h_{i j 1}(\cdot)}{\partial c_{i j}^{* *}}>\frac{\partial h_{i j 2}(\cdot)}{\partial d_{i j}^{* *}}$. Such an inequality rules out, given the concavity of $u(\cdot)$, cases where $d_{i j}$ is too low. ${ }^{29}$ We thus have: $d_{i j}^{* *}>\bar{c}$. Note also that $\frac{\partial h_{i j 1}(\cdot)}{\partial c_{i j}^{* *}}+\pi\left(e_{i j}^{* *}\right)\left[\frac{\partial h_{i j 2}(\cdot)}{\partial c_{i j}^{* *}}-\frac{\partial h_{i j 1}(\cdot)}{\partial c_{i j}^{* *}}\right]=$ $\frac{\partial h_{i j 2}(\cdot)}{\partial d_{i j}^{* *}}$. Hence, given that $\frac{\partial h_{i j 2}(\cdot)}{\partial c_{i j}^{* *}}-\frac{\partial h_{i j 1}(\cdot)}{\partial c_{i j}^{* *}}<0$, it follows that: $\frac{\partial h_{i j 1}(\cdot)}{\partial c_{i j}^{* *}}>$

\footnotetext{
${ }^{28}$ Indeed, in order to obtain an equalization of lifetime welfare between long-lived and short-lived of a given ex ante type, it is necessary, when resources are sufficiently large (i.e. $(4+2 \tilde{\pi}+2 \bar{\pi}) \bar{c} \leq W)$, to fix $d_{i j}^{*}=\bar{c} \forall i, j$.

${ }^{29} \mathrm{By}$ the same argument as in the proof of Proposition 2.
} 
$\frac{\partial h_{i j 2}(\cdot)}{\partial d_{i j}^{* *}}$, implying $c_{i j}^{* *}>d_{i j}^{* *}$, that is, declining consumption profiles along the lifecycle.

Regarding second-period consumption, we also have: $\frac{\partial h_{112}(\cdot)}{\partial d_{11}^{* *}}=\frac{\partial h_{122}(\cdot)}{\partial d_{12}^{* *}}=$ $\frac{\partial h_{212}(\cdot)}{\partial d_{21}^{* *}}=\frac{\partial h_{222}(\cdot)}{\partial d_{22}^{* *}}$. Given the concavity of $h(\cdot)$, and given that patient agents have, ceteris paribus, a higher marginal gain from second-period consumption, we have $d_{2 j}^{* *}>d_{1 j}^{* *}$. The same is true, at the optimum, for effort-loving agents, so that we expect $d_{i 2}^{* *}>d_{i 1}^{* *}$. Hence we have: $d_{22}^{* *}>d_{12}^{* *}, d_{21}^{* *}>d_{11}^{* *}>\bar{c}$.

Let us now compare first-period consumption levels. We have: $\frac{\partial h_{111}(\cdot)}{\partial c_{11}^{* *}}+\pi\left(e_{11}^{* *}\right)\left[\frac{\partial h_{112}(\cdot)}{\partial c_{11}^{* *}}-\frac{\partial h_{111}(\cdot)}{\partial c_{11}^{* *}}\right]=\frac{\partial h_{211}(\cdot)}{\partial c_{21}^{* *}}+\pi\left(e_{21}^{* *}\right)\left[\frac{\partial h_{212}(\cdot)}{\partial c_{21}^{* *}}-\frac{\partial h_{211}(\cdot)}{\partial c_{21}^{* *}}\right]$

Given that $\ell^{*}=1$ and $e_{21}^{* *}>e_{11}^{* *}$, the effect, on the transformed CCPERLE, of a rise in first-period consumption in case of premature death is larger for $\beta_{2}$-type agents than for $\beta_{1}$-type agents. Hence $\frac{\partial h_{111}(\cdot)}{\partial c_{11}^{* *}}<\frac{\partial h_{211}(\cdot)}{\partial c_{21}^{* *}}$. Note that the marginal effect, on the transformed CCPERLE, of a rise in $c_{i j}$ is always larger for short-lived than for long-lived. Thus, the second terms of the LHS and RHS are negative. Moreover, given $d_{21}^{* *}>d_{11}^{* *}$, we expect that the effect, on the transformed CCPERLE, of a rise in first-period consumption in case of long life is larger for $\beta_{1}$-type agents than for $\beta_{2}$-type agents: $\frac{\partial h_{112}(\cdot)}{\partial c_{11}^{* *}}>\frac{\partial h_{212}(\cdot)}{\partial c_{21}^{* *}}$. Thus the first term of the RHS exceeds the first term of the LHS ceteris paribus, and, in absolute value, the factor in brackets of the LHS is larger than the one of the RHS. Note that, as $e_{11}^{* *}<e_{21}^{* *}$, more weight is assigned to the (negative) second term of the RHS than to the (negative) second term on the LHS. Thus the result depends on how large survival chances are.

- If low survival chances, i.e. a low $\pi(\cdot)$ for all effort levels, then we have: $c_{21}^{* *}>c_{11}^{* *}$, on the grounds of the larger welfare loss due to a shorter life in case of patient agents, even though that loss is less likely than for impatient agents, who make less effort.

- If high survival chances, i.e. a high $\pi(\cdot)$ for all effort levels, then we have: $c_{21}^{* *}<c_{11}^{* *}$, on the grounds of the more likely welfare loss for impatient agents, as these make less effort, even though that loss is less sizeable than for patient agents.

Regarding other first-period consumption levels, we have:

$\frac{\partial h_{111}(\cdot)}{\partial c_{11}^{* *}}+\pi\left(e_{11}^{* *}\right)\left[\frac{\partial h_{112}(\cdot)}{\partial c_{11}^{* *}}-\frac{\partial h_{111}(\cdot)}{\partial c_{11}^{* *}}\right]=\frac{\partial h_{121}(\cdot)}{\partial c_{12}^{* *}}+\pi\left(e_{12}^{* *}\right)\left[\frac{\partial h_{122}(\cdot)}{\partial c_{12}^{* *}}-\frac{\partial h_{121}(\cdot)}{\partial c_{12}^{* *}}\right]$

In case of short life, the marginal gain from first period consumption is larger for agents who have, ceteris paribus, the lowest CCPERLE, i.e. agents who like effort. Hence $\frac{\partial h_{111}(\cdot)}{\partial c_{11}^{* *}}<\frac{\partial h_{121}(\cdot)}{\partial c_{12}^{* *}}$ ceteris paribus. In case of long-lived agents, we also have $\frac{\partial h_{112}(\cdot)}{\partial c_{11}^{* *}}<\frac{\partial h_{122}(\cdot)}{\partial c_{12}^{* *}}$. However, given that the marginal effect, on the transformed CCPERLE, of a rise in $c_{i j}$ is always larger for short-lived than for long-lived, the terms in brackets are negative. Note, however, that, as $\frac{\partial h_{111}(\cdot)}{\partial c_{11}^{* *}}<\frac{\partial h_{121}(\cdot)}{\partial c_{12}^{* *}}$ and $\frac{\partial h_{112}(\cdot)}{\partial c_{11}^{* *}}<\frac{\partial h_{122}(\cdot)}{\partial c_{12}^{* *}}$, the two factors in brackets are likely to be very close to each others. Hence, the first terms will drive the optimal allocation of resources at the young age. Given $\frac{\partial h_{111}(\cdot)}{\partial c_{11}^{* *}}<\frac{\partial h_{121}(\cdot)}{\partial c_{12}^{* *}}$, we obtain 
that: $c_{11}^{* *}<c_{12}^{* *}$, since short-lived agents who like effort have larger marginal welfare gains from more consumption, in comparison to effort-averse agents. Following a similar rationale, we obtain also: $c_{21}^{* *}<c_{22}^{* *}$.

\subsection{Proof of Proposition 7}

Here again, the FOCs of the social planner's problem are the same as above, except that $h_{i j 1}\left(c_{i j}, e_{i j}\right) \equiv g \circ f_{i j 1}\left(c_{i j}, e_{i j}\right)$ and $h_{i j 2}\left(c_{i j}, d_{i j}, e_{i j}\right) \equiv g \circ f_{i j 2}\left(c_{i j}, d_{i j}, e_{i j}\right)$, where $f\left(\frac{U_{i j}^{1}}{1+\beta_{i}}\right) \equiv f_{i j 1}\left(c_{i j}, e_{i j}\right)$ and $f\left(\frac{U_{i j}^{2}}{1+\beta_{i}}\right) \equiv f_{i j 2}\left(c_{i j}, d_{i j}, e_{i j}\right)$. 0 .

From the last four FOCs, we obtain, as above: $e_{12}^{* *}=e_{22}^{* *}=\bar{e}>e_{21}^{* *}>e_{11}^{* *}>$

Let us now combine, for a given type $i j$, the FOCs for optimal consumption at the young age and at the old age: $\frac{\partial h_{i j 1}(\cdot)}{\partial c_{i j}^{* *}}-\pi\left(e_{i j}^{* *}\right) \frac{\partial h_{i j j}(\cdot)}{\partial c_{i j}^{* *}}=\frac{\partial h_{i j 2}(\cdot)}{\partial d_{i j}^{* * *}}-$ $\pi\left(e_{i j}^{* *}\right) \frac{\partial h_{i j 2}(\cdot)}{\partial c_{i j}^{* *}}$. Given that $h \equiv g \circ f\left(\frac{U_{i j}^{\ell}}{1+\beta_{i}}\right)$ is concave, we have that $\frac{\partial h_{i j 1}(\cdot)}{\partial c_{i j}^{* *}}>$ $\frac{\partial h_{i j 2}(\cdot)}{\partial c_{i j}^{* *}}$ ceteris paribus. Hence, the above condition requires $\frac{\partial h_{i j 1}(\cdot)}{\partial c_{i j}^{* *}}>\frac{\partial h_{i j 2}(\cdot)}{\partial d_{i j}^{* *}}$. Such an inequality rules out, given the concavity of $u(\cdot)$, cases where $d_{i j}$ is too low. Hence $d_{i j}^{* *}>\bar{c} .^{30}$

Note also that $\frac{\partial h_{i j 1}(\cdot)}{\partial c_{i j}^{* *}}+\pi\left(e_{i j}^{* *}\right)\left[\frac{\partial h_{i j 2}(\cdot)}{\partial c_{i j}^{* *}}-\frac{\partial h_{i j 1}(\cdot)}{\partial c_{i j}^{* *}}\right]=\frac{\partial h_{i j 2}(\cdot)}{\partial d_{i j}^{* * *}}$. Hence, given that $\frac{\partial h_{i j 2}(\cdot)}{\partial c_{i j}^{* *}}-\frac{\partial h_{i j 1}(\cdot)}{\partial c_{i j}^{* *}}<0$, it follows that: $\frac{\partial h_{i j 1}(\cdot)}{\partial c_{i j}^{* *}}>\frac{\partial h_{i j 2}(\cdot)}{\partial d_{i j}^{* *}}$, implying $c_{i j}^{* *}>d_{i j}^{* *}$, that is, declining consumption profiles along the lifecycle.

Regarding second-period consumption, we also have: $\frac{\partial h_{112}(\cdot)}{\partial d_{11}^{* * *}}=\frac{\partial h_{122}(\cdot)}{\partial d_{12}^{* *}}=$ $\frac{\partial h_{212}(\cdot)}{\partial d_{21}^{* *}}=\frac{\partial h_{222}(\cdot)}{\partial d_{22}^{* *}}$. Here again, given the concavity of $h(\cdot)$, and given that patient agents have, ceteris paribus, a higher marginal gain from second-period consumption, we expect $d_{2 j}^{* *}>d_{1 j}^{* *}$. The same is true, at the optimum, for effortloving agents, so that we expect $d_{i 2}^{* *}>d_{i 1}^{* *}$. Hence we have: $d_{22}^{* *}>d_{12}^{* *}, d_{21}^{* *}>$ $d_{11}^{* *}>\bar{c}$.

Let us now compare first-period consumption levels. We have:

$\frac{\partial h_{111}(\cdot)}{\partial c_{11}^{* *}}+\pi\left(e_{11}^{* *}\right)\left[\frac{\partial h_{112}(\cdot)}{\partial c_{11}^{* *}}-\frac{\partial h_{111}(\cdot)}{\partial c_{11}^{* *}}\right]=\frac{\partial h_{211}(\cdot)}{\partial c_{21}^{* *}}+\pi\left(e_{21}^{* *}\right)\left[\frac{\partial h_{212}(\cdot)}{\partial c_{21}^{* *}}-\frac{\partial h_{211}(\cdot)}{\partial c_{21}^{* *}}\right]$

Given that $\ell^{*}=2$ and $e_{21}^{* *}>e_{11}^{* *}$, the effect, on the transformed CCPERLE, of a rise in first-period consumption in case of premature death is larger for $\beta_{2}$-type agents than for $\beta_{1}$-type agents. Hence $\frac{\partial h_{111}(\cdot)}{\partial c_{11}^{* *}}<\frac{\partial h_{211}(\cdot)}{\partial c_{21}^{* *}}$. Note that the marginal effect, on the transformed CCPERLE, of a rise in $c_{i j}$ is always larger for short-lived than for long-lived. Thus, the second terms of the LHS and RHS are negative. Moreover, given $d_{21}^{* *}>d_{11}^{* *}$, we expect that the effect, on the transformed CCPERLE, of a rise in first-period consumption in case of long life is larger for $\beta_{1}$-type agents than for $\beta_{2}$-type agents: $\frac{\partial h_{112}(\cdot)}{\partial c_{11}^{* *}}>\frac{\partial h_{212}(\cdot)}{\partial c_{21}^{* *}}$. Thus the first term of the RHS exceeds the first term of the LHS ceteris paribus, and, in absolute value, the factor in brackets of the LHS is larger than the one of the RHS. Note that, as $e_{11}^{* *}<e_{21}^{* *}$, more weight is assigned to the (negative)

${ }^{30}$ Same argument as in the proof of Proposition 2. 
second term of the RHS than to the (negative) second term on the LHS. Thus the result depends on how large survival chances are.

- If low survival chances, i.e. a low $\pi(\cdot)$ for all effort levels, then we have: $c_{21}^{* *}>c_{11}^{* *}$, on the grounds of the larger welfare loss due to a shorter life in case of patient agents, even though that loss is less likely than for impatient agents, who make less effort.

- If high survival chances, i.e. a high $\pi(\cdot)$ for all effort levels, then we have: $c_{21}^{* *}<c_{11}^{* *}$, on the grounds of the more likely welfare loss for impatient agents, as these make less effort, even though that loss is less sizeable than for patient agents.

Regarding other first-period consumption levels, we have:

$\frac{\partial h_{111}(\cdot)}{\partial c_{11}^{* *}}+\pi\left(e_{11}^{* *}\right)\left[\frac{\partial h_{112}(\cdot)}{\partial c_{11}^{* *}}-\frac{\partial h_{111}(\cdot)}{\partial c_{11}^{* *}}\right]=\frac{\partial h_{121}(\cdot)}{\partial c_{12}^{* *}}+\pi\left(e_{12}^{* *}\right)\left[\frac{\partial h_{122}(\cdot)}{\partial c_{12}^{* *}}-\frac{\partial h_{121}(\cdot)}{\partial c_{12}^{* *}}\right]$

In case of short life, the marginal gain from first period consumption is larger for agents who have, ceteris paribus, the lowest CCPERLE, i.e. agents who dislike effort. Hence $\frac{\partial h_{111}(\cdot)}{\partial c_{11}^{* *}}>\frac{\partial h_{121}(\cdot)}{\partial c_{12}^{* *}}$ ceteris paribus. In case of long-lived agents, we also have $\frac{\partial h_{112}(\cdot)}{\partial c_{11}^{* *}}>\frac{\partial h_{122}(\cdot)}{\partial c_{12}^{* *}}$. However, given that the marginal effect, on the transformed CCPERLE, of a rise in $c_{i j}$ is always larger for short-lived than for long-lived, the terms in brackets are negative. Note, however, that, as $\frac{\partial h_{111}(\cdot)}{\partial c_{11}^{* *}}>\frac{\partial h_{121}(\cdot)}{\partial c_{12}^{* *}}$ and $\frac{\partial h_{112}(\cdot)}{\partial c_{11}^{* *}}>\frac{\partial h_{122}(\cdot)}{\partial c_{12}^{* *}}$, the two factors in brackets are likely to be very close to each others. Hence, the first terms will drive the optimal allocation of resources at the young age. Given $\frac{\partial h_{111}(\cdot)}{\partial c_{11}^{* *}}>\frac{\partial h_{121}(\cdot)}{\partial c_{12}^{* *}}$, we obtain that: $c_{11}^{* *}>c_{12}^{* *}$, since short-lived agents who dislike effort have larger marginal welfare gains from more consumption, in comparison to effort-loving agents. Following a similar rationale, we obtain also: $c_{21}^{* *}>c_{22}^{* *}$. 